\title{
Randomly distributed K14+ breast tumor cells polarize to the leading edge and guide collective migration in response to chemical and mechanical environmental cues
}

\author{
Priscilla Y Hwang ${ }^{1,3}$, Audrey Brenot ${ }^{1,3}$, Ashley C King ${ }^{1,3}$, Gregory D. Longmore ${ }^{1,2,3,{ }^{*}}$, and \\ Steven C George $\mathrm{e}^{4}$, \\ 1Department of Medicine (Oncology), Washington Unviersity in St Louis, St Louis MO. \\ ${ }^{2}$ Cell Biology and Physiology, Washington Unviersity in St Louis, St Louis MO. \\ ${ }^{3}$ ICCE Institute, Washington Unviersity in St Louis, St Louis MO. \\ ${ }^{4}$ Department of Biomedical Engineering, University of California, Davis, Davis CA
}

\begin{abstract}
Collective cell migration is an adaptive, coordinated interactive process involving cell-cell and cell-extracellular matrix (ECM) microenvironmental interactions. A critical aspect of collective migration is the sensing and establishment of directional movement. It has been proposed that a subgroup of cells known as leader cells localize at the front edge of a collectively migrating cluster and are responsible for directing migration. However, it is unknown how and when leader cells arrive at the front edge and what environmental cues dictate leader cell development and behavior. Here we addressed these questions by combining a microfluidic device design that mimics multiple tumor microenvironmental cues concurrently with biologically relevant primary, heterogeneous tumor cell organoids. Prior to migration, breast tumor leader cells (K14+) were present throughout a tumor organoid and migrated (polarized) to the leading edge in response to biochemical and biomechanical cues. Impairment of either CXCR4 (biochemical responsive) or the collagen receptor DDR2 (biomechanical responsive) abrogated polarization of leader cells and directed collective migration. This work demonstrates that K14+ leader cells utilize both chemical and mechanical cues from the microenvironment to polarize to the leading edge of collectively migrating tumors.
\end{abstract}

\section{Keywords}

chemoattractant; oxygen tension; organ-on-a-chip; interstitial flow; collective migration; leader cells

\footnotetext{
Corresponding Authors: Greg Longmore, Washington University Schooll of Medicine, ICCE Institute, BJCIH $7^{\text {th }}$ floor, $425 \mathrm{~S}$. Euclid Avenue, St. Louis MO 63110, Ph: 314-362-8834, glongmore@ wustl.edu; Steven C George, University of California, Davis, 451 E. Health Sciences Drive, room 2311, Davis, CA 95616, Ph: 530-752-9978, scgeorge@ ucdavis.edu. *o-senior authors
} 


\section{Introduction}

Collective migration, the process by which groups of cells migrate in a coordinated fashion, is essential for normal development yet also contributes to disease, such as during cancer metastasis $[1,2]$. Metastases were thought to occurr predominantly through individual cell dissemination from the primary tumor to enter the blood stream and lymphatics to target organs. However, recent studies of tumor invasion and migration patterns suggest that a large number of solid tumors also invade and migrate as multicellular units (collective migration), including in the blood stream [3, 4]. To move collectively, as opposed to single cell migration, requires coordinated cell-cell and cell-matrix interactions $[5,6]$ that can also impact tumor response to therapies. The morphological organization of collectively migrating tumor cells in vivo includes an array of patterns ranging from strands of cells that emanate from tumors and "break off" to clusters of cells within the surrounding ECM [7, 8]. Much of our understanding of single cell and collective migration derives from in vitro models $[9,10]$. In this study, we establish a novel in vitro model of collective migration using primary tumor-derived organoids.

During collective migration, directional cell movements are interdependent and coordinated through stable or transient cell-cell and cell-extracellular matrix (ECM) contacts. Prior studies suggest different roles for cells within the collectively migrating cluster; specifically, leader and follower cells. Leader cells are located at the leading edge or front of the collective unit and potentially detect and transduce environmental guidance cues that control the direction of migration. It is still largely unknown, however, what characteristics classify a leader cell, thus most studies of leader cell studies are limited to investigating phenotypic differences for the cells located at the front edge after collective migration has initiated. Studies in mouse breast cancer models, primary breast tumor organoids in culture, and correlative human histologic studies reveal that keratin $14\left(\mathrm{~K}_{1} 4^{+}\right)$epithelial-derived tumor cells are present at the leading edge of invasive tumor aggregates, and have thus been coined leader cells $[3,10,11]$. How these leader cells develop and arrive at the front edge, and whether this phenomenon is necessary and sufficient to effect directed collective migration is largely unknown.

Several hypotheses have been proposed regarding leader cell development. In one, all cells within a collective cluster have the potential to become leader cells, and leader cell development is due to phenotypic switches for cells at the edge in response to specific and localized environmental cues. Alternatively, a subset of specialized cells within the collective cluster with the potential to be leader cells move to the leading edge and there direct collective migration $[8,12,13]$. In vivo, studies of these questions suffer from limited temporal and spatial resolution to probe cellular and molecular events, such as leader cell development [14, 15]. And, to date, in vitro models have generally focused on the response of aggregated homogeneous tumor cell lines to single microenvironmental cues such as a soluble factor(s) [16-18], neighboring cells (e.g., fibroblast) [19, 20], or a defined extracellular matrix [21-23]. This approach is limited in its capacity to truly mimic in vivo conditions, largely because tumors clusters are composed of heterogeneous cell populations and even individual cell types within invasive tumor clusters display dramatic phenotypic plasticity during the progression to metastasis [24-27]. 
Here we present a transparent 3D microfluidic system that allows for dynamic real time imaging and the establishment of multiple environmental stimuli concurrently. In this device we place primary, heterogeneous breast tumor organoids isolated from genetically defined spontaneous mouse tumor models to investigate leader cell development and directed collective migration. By combining microfluidic technology and K14-GFP labeled leader cells in primary breast tumor organoids, we can resolve competing hypotheses regarding leader cell development. Our study reveals that randomly distributed pre-existing $\mathrm{K} 14^{+}$ leader cells migrate through the organoid to "polarize" to the front edge in response to multiple dynamic changes in the tumor microenvironment, specifically chemokine gradients and interstitial fluid flow. Furthermore, our study reveals a previously unknown sensitivity of K14-leader cell polarization to the front edge and directed collective migration to signaling through the SDF-1 chemokine receptor CXCR4 and the fibrillar collagen receptor DDR2. This work demonstrates the feasibility of engineering a pathophysiological in vitro tumor microenvironment model system that can provide high spatial resolution to investigate dynamic events of primary cancer progression.

\section{Materials and Methods}

\section{Microfluidic device fabrication and performance}

Microfluidic devices were synthesized using soft lithography techniques and cast in polydimethylsiloxane (PDMS), as previously described [28]. We confirmed the ability to establish and maintain an SDF1 gradient for 24 hours using COMSOL, and experimental delivery of $8 \mathrm{kDa}$-FITC-dextran (similar weight to SDF1).

\section{Extracellular Matrix}

Collagen I (rat tail, Trevigen; 1-4 mg/ml) was used to model the extracellular matrix. Mechanial properties were measured via oscillatory shear testing (AR200 Rheometer,E, Pa). We measured the average fiber diameter of the hydrogels with immunostaining and second harmonic (SHG) imaging (Zeiss).

\section{Mice tumor organoid isolation and culture}

MMTV-PyMT mice were obtained from Jackson laboratory and crossed to K14-GFP-actin mice (transgenic mouse in which GFP-actin fusion protein was expressed under the control of the keratin-14 promoter, which is functional in mitotically active epidermal cells [29]) to generate K14-GFP tagged MMTV-PyMT mice. They express EGFP-Actin only in K14 +ve cells. The endogenous K14 gene is not altered in these mice. We refer to all K14 positive cells obtained from this mouse as "K14-GFP". Ubiquitous Ddr2-/-; MMTV-PyMT mice were generated as previously described [30]. MMTV-PyMT; Ddr2+/-; K14-GFP mice were crossed with Ddr2+/-, K14-GFP mice to generate MMTV-PyMT; Ddr2-/-; K14-GFP mice. Tumor bearing mice were monitored weekly and euthanized at 12 weeks. All mice were used in compliance with the Washington University Institutional Animal Care and Use Committee and approved under protocol \#20150145.

Mice mammary tumor organoids were obtained as previously described [9], mixed with 2 $\mathrm{mg} / \mathrm{ml}$ collagen I solution, loaded into the middle tissue chamber of the microfluidic device, 
allowed to polymerize $\left(37^{\circ} \mathrm{C}, 20 \% \mathrm{O}_{2}\right)$, and media (DMEM, $10 \% \mathrm{FBS}, \mathrm{P} / \mathrm{S}$ ) was delivered to the top and bottom fluidic lines and cultured in $5 \% \mathrm{O}_{2}$. We also delivered various concentrations of SDF1(Sigma-Aldrich) to the top and bottom fluidic lines to establish a spectrum of SDF1 gradients. Finally, we induced a physiological level of interstitial fluid flow $(12 \mu \mathrm{m} / \mathrm{sec}[28])$.

\section{Live-cell Imaging and analysis}

After culturing organoids for 48 hours in $5 \% \mathrm{O}_{2}$, we induced biochemical or biomechanical stimuli and performed live-cell imaging (Nikon Ti-E, 10x, 40x, 63x; controlled temperature, humidity, and oxygen $\left(5 \% \mathrm{O}_{2}\right)$ ). Each organoid within the device was marked using Metamorph or Nikon Imaging software and pictures were taken every 20 minutes for a maximum of 18 hours. After imaging, devices were used for immunofluorescence labeling and imaging, or organoids were extracted from the device for gene expression studies.

Image analysis was performed using Metamorph, Matlab, and FIJI to quantify organoid migration efficiency $(\%)$ in the direction of the gradient, average velocity $(\mu \mathrm{m} / \mathrm{min})$, and direction of travel. Migration efficiency is defined as follows, where $\mathrm{y}$ and $\mathrm{x}$ are the coordinates for the final location of the organoid with respect to the beginning (origin) location:

$$
\text { Migration efficiency }(\%)=\frac{\tan ^{-1}\left(\frac{y}{|x|}\right)}{90^{\circ}} \times 100 \%
$$

This definition provides an efficiency of $+/-100 \%$ for migration that is parallel to the $y$ axis; a positive value is the direction of the spatial morphogen (SDF1) gradient or in the direction of interstitial flow.

We also tracked and quantified K14-GFP localization over time. At various time points, images of organoids were divided into top (front; direction of migration) and bottom (back) halves, and total K14-GFP fluorescence of each half was calculated using FIJI and the following formula: cell fluorescence $=$ integrated density $-($ area of half $\times$ mean fluorescence of background).

\section{Immunofluorescence and analysis}

All immunostaining was performed after imaging studies with organoids maintained within the devices, and all reagents were delivered via microfluidic lines. After fixing and blocking, organoids were stained for CXCR4 (Abcam), DDR2 (Abcam) and K14 (Abcam); all primary antibody staining was incubated overnight at 4C. Species-specific secondary antibodies (488 or 566 wavelength) and nuclei staining (DAPI) were also used. Imaging was performed via confocal microscopy (Zeiss, 63X). Analysis was performed using FIJI to quantify fluorescence intensity and localization. Fluorescence was calculated in the same manner as K14-GFP localization (described above). 


\section{Gene expression}

Organoids were extracted for gene expression studies after live-cell imaging. Organoids were extracted and lysed within the device by delivering RLT lysis buffer (Qiagen RNeasy plus Micro Kit) with $\beta$-mercaptoenthanol directly to the tissue chamber. Cell lysis was collected and mRNA was extracted using RNEasy Plus Micro kit (Qiagen, protocol supplied by manufacturer). cDNA was synthesized using a High-Capacity cDNA Reverse Transcription Kit (Applied Biosystems, protocol supplied by manufacturer), and qRT-PCR was performed using Taqman probes (Invitrogen) for CXCR4, K14, DDR2, and E-cadherin with GAPDH or $18 \mathrm{~s}$.

\section{CXCR4 inhibition and knockdown}

In order to inhibit CXCR4, we delivered CXCR4 inhibitor AMD3100 (EMD Millipore) to organoids via microfluidic lines every 24 hours during the culture period. For knockdown studies, we synthesized two different shRNA lentivirus particles specific for CXCR4 (Origene, TL500383, sequence A and D) with a GFP tag. We transduced organoids directly in the microfluidic devices via fluidic lines. Organoids were exposed to transduction media for 16 hours, and successful transduction was confirmed by visualizing GFP expression. Organoids began to express GFP after 48 hours, and at that time point, we transferred devices to $5 \% \mathrm{O}_{2}$ for the remainder of the culture period. Successful knockdown of CXCR4 was confirmed via qRT-PCR quantification.

\section{DDR2 inhibition and knockout}

We delivered WRG-28 [31], an allosteric, selective small molecule DDR2 inhibitor, to organoids via microfluidic lines every 24 hours of culture period. We also isolated tumor organoids from ubiquitous MMTV-PyMT; Ddr2 ${ }^{-/}$; K14-GFP mice.

\section{Statistics}

All data respresent mean \pm SEM of the indicated number of experiments. Statistical analysis (JMP software) was performed using ANOVA with Tukey's post-hoc anlaysis, considering $\mathrm{p}<0.05$ as statistically significant.

See also Supplemental methods for extended details about:

Organoid generation and embedding in microfluidic devices

Immunofluorescence of organoids in microfluidic devices

Gene expression analysis in organoids

CXCR4 shRNA depletion in organoids within the microfluidic devices

\section{Results}

\section{A microfluidic system to investigate 3D collective migration}

Limiting our understanding of collective tumor cell migration are pathophysiologically relevant ex vivo model systems that allow for imaging this phenomenon at high spatial and 
temporal resolution. To address this problem we adapted a previously designed transparent microfluidic device [28] with the capability to manipulate the chemical and physical microenvironment, concurrently, so as to observe how genetically defined primary breast tumor organoids move through 3D collagen I hydrogels (Fig. 1A). The design includes three parallel tissue chambers surrounded by two parallel microfluidic lines that deliver the necessary media to the tissue chamber via diffusion or interstitial flow [28]. The number of ports surrounding each of the tissue chambers were optimized such that, when the pressure in the outer microfluidic lines is equivalent, one can deliver the desired chemokine in the top microfluidic line to establish a near linear and constant chemokine gradient [28]. We confirmed the ability to form and maintain a chemokine gradient (Fig. 1B): 8 kDa FITCDextran (similar molecular weight to SDF1) was placed in the top chamber and a near linear gradient rapidly ( $<2 \mathrm{hr}$ ) formed and was maintained for 24 hours (Fig. 1B). The experimental result was consistent with a computational model (COMSOL) of mass transport (Fig. 1B-C). We confirmed that a tumor cell line, the invasive breast cancer cell line MDA-MB-231, was responsive to an SDF-1 chemokine gradient in these devices (Sup Fig. 1).

\section{Primary breast tumor organoids in vitro respond in a similar manner to in vivo conditions}

As a pathophysiologically relevant source of tumor cells to study collective migration we chose to analyze primary breast tumor organoids from genetically defined spontaneous MMTV-PYMT mouse models of breast cancer. The MMTV-PyMT breast tumor model is highly invasive and metastatic. Breast tumor organoids, like in vivo tumors, were composed of a heterogeneoius mix of tumor cells (K14 and K8) and also included some non-tumor stromal cells, such as cancer associated fibroblasts (CAFs) and CD45+ leukocytes (Fig 2A; Sup Fig 2). These tumor organoids that average $200-500$ cells were placed into the microfluidic device immediately following isolation (i.e., never cultured on plastic).

In vivo, most tumors exist in a hypoxic environment that can influence expression of several proteins involved with migration [17, 32], such as CXCR4 (the receptor for the chemokine SDF1) and the collagen receptor DDR2 [30,33-36]. We confirmed that primary mouse breast tumor organoids increased gene and protein expression of CXCR4 and DDR2 when cultured under low oxygen (5\% or less) in our microfluidic device compared to high oxygen (20\%) (Fig. 2B-D). Prior to exposure to hypoxia, the distribution of putative K14+ leader cells cells throughout the organoid was random (Fig. 2B-C). Following exposure to low oxygen ( $48 \mathrm{hrs}$ ) there was no change in the number of K14 cells, the intensity of K14 immunofluorescent staining, the distribution (random) of K14 cells, or K14 gene expression within tumor organoids (Fig. 2B-D).

These observations were confirmed in vivo using mouse PyMT and human invasive breast tumors samples. In breast tumors K14 cells were present predominantly at the tumor stromal boundary (Fig. 2E). CXCR4 expression was increased in tumors and present in K14+ cells although non-K14 tumor cells also expressed CXCR4 (Fig. 2E, F). The spatial distribution of SDF1, the ligand for CXCR4, was highly heterogeneous throughout the tumor tissue (Fig. 2E, F). DDR2 expression also increased in invasive breast tumors as expected [34] and was localized to cells at the invasive leading edge, in a similar pattern to K14 expressing cells 
(Fig. 2E, F). Quantitative PCR analysis of mouse PyMT tumors versus normal mammary gland tissue revealed that both CXCR4 and DDR2 mRNA levels increased while K14 mRNA levels were unchanged (Fig. 2G). Analysis of human breast tumor microarrays [37] was consistent with findings in mouse tumors: no differences were observed for K14 gene expression between tumor and non-tumor tissue but there were significant increases in tumor tissue CXCR4 and DDR2 (3.08x and 2.65x respectively) (Fig. 2H; analysis performed with publically available microarray data [37]).

Tumor organoids exposed to hypoxia alone did not collectively migrate in a directional manner but did move randomly (Fig. 3). Since hypoxia alone did not induce directed collective migration, but did activate expression of CXCR4 in K14 cells, we asked whether exposure of tumor organoids to the CXCR4 ligand, a SDF-1 chemokine gradient (+ hypoxia) would induce directional migration. When tumor organoids under hypoxic conditions were exposed to a SDF-1 gradient ("Gradient") for 16h, directed migration occurred in the direction of the positive gradient (i.e., towards the higher concentration) with a higher average migration velocity (Fig. 3A-D; Movie 1). We refer to this spatially guided migration as "directed collective migration" and distinguish it from random collective migration that produces no net migration in any particular direction. Furthermore, we observed differences in the distribution of K14 cells within tumor organoids. When tumor organoids migrated in a directed collective manner, K14 expressing cells were localized at the front edge, in the direction of migration (Fig. 3A). When tumor organoids did not migrate, K14 cells remained randomly distributed throughout the organoid. Tumor organoids exposed to uniform SDF1 ("No Gradient") at low oxygen did not directionaly migrate but did undergo random collective migration with a velocity greater than in hypoxia alone (Fig. 3D). The average random velocity of organoid migration was not different in organoids exposed to a gradient versus non-gradient of SDF1 (Fig. 3D). Under low oxygen and a SDF1 gradients, the entire mass of cells migrated as a collective unit, rather than leading invasive strands of cells. Under high oxygen $\left(20 \% \mathrm{O}_{2}\right)$ and a SDF-1 gradient, no directional migration was observed (Sup Fig 2).

\section{K14 leader cells migrate within or through organoids to the leading edge (polarize) and guide collective migration in response to microenvironmental cues}

There are at least two possibilities as to how K14 cells polarize to the leading edge of invasive tumors. One, in response to signals from the surrounding tumor ECM cells at the leading edge undergo a phenotypic conversion to form K14 cells [9]. Alternatively, as we observed herein, pre-existing randomly distributed K14 cells actively polarize to the leading edge in response to chemokine gradients and possibly other tumor ECM signals. To distinguish between these two possibilities, we generated K14-GFP; MMTV-PyMT mice (as described in Methods). These mice express a GFP-actin fusion protein in K14 cells that allow one to monitor K14 cells in real time (videos). Hereafter, we refer to these genetically labeled cells as K14-GFP cells, as opposed to immunostained K14 cells. Tumor organoids were isolated and placed in the central chamber of the microfluidic device, exposed to hypoxia and SDF1 gradient, and time lapse videos of organoid migration obtained and analyzed. In static images taken from these movies, K14-GFP cells were initially randomly distributed throughout the tumor organoids (Fig. 3E). After exposure to an SDF1 gradient, 
K14-GFP cells, regardless of their original position, appeared to actively migrate to and accumulate (i.e., polarize) at the edge of the organoid exposed to the highest concentration of SDF1 (Fig. 3E, quantified in 3F; see also Movie 2). This movement occurred over 6-12h. Without exposure to the SDF1 gradient, K14-GFP cells did not migrate (Fig. 3E, lower panels). Importantly, the total level of K14-GFP fluorescence did not change during the course of any of these experiments (average fold change between the beginning and the end of the imaging period $=1.1 \pm 0.23$ ).

These live cell videos demonstrated that pre-existing, but randomly distributed, K14 cells actively migrate (polarize) to what will become the leading edge of collectively migrating tumor organoids.

\section{K14 polarization and directed collective migration require both CXCR4 and DDR2}

How K14 cells polarize to the leading edge of migrating tumor clusters and whether this is required for directed collective migration, as well as the environmental and cell intrinsic signals controlling K14 cell functions during breast tumor collective migration are largely unknown. We first asked whether the SDF1-CXCR4 signaling was critical for K14 cell functions in directed collective cell migration. To do so, we employed a series of genetic and pharmacologic studies. CXCR4 expression was depleted in in all cells within primary tumor organoids by transduction with multiple lentiviruses expressing shRNAs targeting CXCR4. The shRNA-expressing lentiviruses also expressed GFP in infected cells that allowed for confirmation of successful transduction (Sup Fig. 3). Successful mRNA depletion was confirmed by qRT-PCR (Sup Fig. 3). In an SDF1 gradient, CXCR4 depleted organoids did not migrate in a directional manner and average random velocity was also reduced (Fig. 4AC). Similar results were observed when WT organoids were inhibited with the CXCR4 inhibitor AMD3100 (Fig. 4D-E). In CXCR4 inhibited organoids, K14 cells failed to polarize to a leading edge (Fig. 4F).

The action of the fibrillar collagen receptor DDR2 in K14 breast tumor cells has been implicated as controlling tumor cell migration in culture systems and metastasis in vivo [30]. To determine if the action of DDR2 in breast tumor organoids was required for directed collective migration, and if so how, we isolated primary PyMT breast tumor organoids from ubiquitous Ddr2 $2^{-/}$mice or treated WT tumor organoids with a small molecule inhibitor of DDR2, WRG-28 [31]. In an SDF1 gradient (+ hypoxia), both WRG-28 treated and Ddr2-/organoids failed to migrate in a directed manner, exhibited significantly slower average velocity than wild type controls, and K14 cells did not polarize to a leading edge (Fig. 4DF).

Finally, we asked whether CXCR4 or DDR2 play a role in K14 leader cell polarization using K14-GFP-Actin expressing tumor organoids and real time imaging. When CXCR4 was inhibited with AMD3100, there was no change in localization of K14-GFP cells throughout the live-cell imaging period even with exposure to the SDF1 gradient (Fig. 4G). Primary breast tumor organoids from Ddr2-/-; K14-GFP-Actin; MMTV-PyMT mice did not migrate, and K14-GFP cells did not polarize to a leading edge (Fig. 4G and Movie 3). 
In sum, these results indicated that the action of both CXCR4 and DDR2 in tumor cells and possibly other non-tumor stromal cells within breast tumor organoids were required for K14 cell polarization and directed collective migration in response to an SDF1 chemotactic gradient under hypoxic conditions.

\section{Chemokine gradient shape and magnitude dictates collective migration}

The distribution of SDF1, the ligand for CXCR4, within tumor tissue was heterogeneous (Fig. 2E), suggesting the possibility that different spatial gradients of SDF1 (magnitude and direction) may exist within the tumor microenvironment and influence K14 cell polarization and directed collective migration. Therefore, we asked whether there was an optimal SDF1 gradient that induced directed collective migration. To test this, we exposed MMTV-PyMT breast tumor organoids to a series of different fixed linear gradients of SDF1 under hypoxic conditions (Fig. 5A). We tested 7 different combinations of three different mean concentrations $(25,50$, and $12.5 \mathrm{ng} / \mu \mathrm{l})$ and three different gradient magnitudes (difference in the concentration of SDF1 across the device divided by the total length of the device: 0.167 ng. $\mu \mathrm{l}^{-1} \mu \mathrm{m}^{-1}, 0.083 \mathrm{ng} . \mu \mathrm{l}^{-1} \cdot \mu \mathrm{m}^{-1}, 0.042 \mathrm{ng} . \mu \mathrm{l}^{-1} \cdot \mu \mathrm{m}^{-1}$ ) (Fig. 5A). By varying the mean concentration and gradient magnitude, we found that there existed both a minimum and maximum concentration and gradient magnitude of SDF1 required for directed collective migration to occur. Directed collective migration (migration efficiency and migration velocity was $90 \% \pm 7.07 \%$ and $0.42 \pm 0.072 \mu \mathrm{m} / \mathrm{min}$, respectively) was observed throughout the entire imaging period for group 50_0 where the mean concentration was $25 \mathrm{ng} / \mathrm{ul}$ with a gradient magnitude of $0.083 \mathrm{ng} / \mathrm{um}$ (Fig. 5B-D). When the mean concentration was held constant (at $25 \mathrm{ng} / \mu \mathrm{l}$ ) but the gradient was decreased to $0.042 \mathrm{ng} \cdot \mu \mathrm{l}^{-1} \mu_{\mathrm{m}}^{-1}$ (group 37.5_12.5), migration velocity and efficiency were both decreased. If the gradient magnitude was held constant $\left(0.042 \mathrm{ng} . \mu \mathrm{l}^{-1} \cdot \mu \mathrm{m}^{-1}\right.$; groups 37.5_12.5 and 25_0) but mean concentration decreased from 25 to $12.5 \mathrm{ng} . \mu \mathrm{l}^{-1}$, migration efficiency and velocity were both abrogated. While both migration efficiency and velocity were positive functions of the mean concentration and the gradient magnitude, it was also clear that this phenomenon was saturable: the maximum mean concentration and gradient (50 ng. $\mu \mathrm{l}^{-1}$ and $0.167 \mathrm{ng} \cdot \mu \mathrm{l}^{-1} \cdot \mu \mathrm{m}$ ${ }^{-1}$ for group 100_0) produced zero migration efficiency while maintaining the migration velocity.

We also asked whether exposure to chemokine gradients impact the localization of K14 cells within tumor organoids. To answer this, we quantified K14 expression and K14 cell localization at the beginning and end of each experiment. In a SDF1 gradient where directed collective migration occured (group 50_0), initially randomly distributed K14 cells localized to the leading edge of the collective group at the end (Fig. 5E) with no apparent change in the number of K14 cells or intensity of K14 staining per cell (Fig. 5B). This polarization response of K14 cells only occurred in gradients that supported/facilitated sustained directed migration (e.g., group 50_0) (Fig. 5E). In all other conditions K14 cells remained randomly distributed throughout the tumor organoid at the end of the experiment (Fig. 5E). A similar pattern of polarization was observed for CXCR4 expressing cells (Fig. 5F). Indeed, Pearson's co-localization coefficient indicated that the extent of CXCR4 and K14 colocalization was highest in directed collectively migrating tumor organoids (Fig. 5G). Two conditions: $75 \_25$ and $37.5 \_12.5$, produced positive directed migration and velocity but at 
the end of the experiment we noted that K14 cells had not polarized to the leading edge. When the migration pattern of these two conditions was subdivided into the first six hours, and time $>6$ hours it was found that positive directed migration efficiency and velocity only occurred during the first six hours (Fig. 5H).

In summary, omly in hypoxia and chemokine gradients that supported sustained directed collective migration and velocity did K14 cells polarize to the leading edge (Fig. 5B, Sup Fig 4). This suggested that polarization of K14 cells within tumor organoids was required for directed collective migration.

\section{Collagen hydrogel properties alter the ability for collective migration but not K14 polarization}

Matrix architecture and mechanical properties impact individual and collective cell migration of homogeneous tumor cell lines. To determine if and how matrix composition and stiffness impact K14 leader cell polarization and collective migration of tumor organoids, we synthesized, characterized and encapsulated tumor organoids in collagen hydrogels of varying stiffness and fiber diameter (Sup Fig. 5): $1 \mathrm{mg} / \mathrm{ml}(\mathrm{E}=20-28 \mathrm{~Pa}$, radius $=0.1-0.7 \mu \mathrm{m}), 2 \mathrm{mg} / \mathrm{ml}(\mathrm{E}=55-63 \mathrm{~Pa}$, radius $=0.1-0.5 \mu \mathrm{m})$, and $4 \mathrm{mg} / \mathrm{ml}(\mathrm{E}=150-172 \mathrm{~Pa}$, radius $=0.01-0.2 \mu \mathrm{m}$ ). Time lapse videos of K14-GFP-Actin; MMTV-PyMT tumor organoids exposed to hypoxia and a SDF1 gradient demonstrated that tumor organoids were unable to collectively migrate in softer $(1 \mathrm{mg} / \mathrm{ml})$ and stiffer $(4 \mathrm{mg} / \mathrm{ml})$ collagen matrices (Fig. 6A-D). Despite this, K14-GFP cells still polarized to the front edge in all conditions (Fig. 6D). Collagen fibers were remodeled during directed collective migration as SHG imaging of tumor organoids in $2 \mathrm{mg} / \mathrm{ml}$ collagen hydrogel exposed to SDF1 chemokine gradient compared to "no SDF1" control (Fig. 6B) revealed prominent collagen fiber alignment and thickening in the direction of migration. In organoids that did not migrate (e.g., CXCR4 or DDR2 inhibited or genetically deleted) collagen fibers remained disperse without alignment or thickening.

These findings suggested that the collagen matrix environment impacts directed collective migration of tumor organoids. Changes in collagen content influenced both K14 polarization and directed collective migration. Manipulating the collagen matrix also revealed that K14 cell polarization alone was not sufficient for directed collective migration to occur.

\section{K14 leader cells polarize during collective migration in the direction of interstitial fluid flow and this requires Ddr2}

Interstitial fluid flow within tumors is an environmental biomechanical cue that can impact cell migration. Many different patterns of interstitial fluid flow exist in vivo, but it is difficult to reliably study how fluid flow impacts collective migration in vivo due to the inability to measure fluid flow and quantify collective migration features simultaneously. Using our microfluidic device we were able to generate interstitial fluid flow gradients, quantify fluid flow, and then measure their impact upon directed collective migration in real-time using primary tumor organoids. In response to fluid flow under low oxygen conditions, collective migration occurred with flow over a period of 12 hours (Fig. 7A-E) with average velocities similar to the migration velocity for organoids exposed to a SDF1 gradient (Fig. 7D). Time- 
lapse videos analyzing K14-GFP cells revealed that they polarized to the front edge in response to fluid flow (Fig. 7A,E). SHG imaging indicated that active remodeling of collagen fibers occured in the direction of migration (Fig. 7B). Analysis of K14-GFP-Actin; $\mathrm{Ddr} 2^{-/}$tumor organoids revealed that the action of DDR2 within tumor cells or other nontumor stromal cells within organoids was required for the polarization of K14 cells to the leading edge and directed collective migration in response to fluid flow (Fig. 7A,E).

\section{Discussion}

In this study, we investigated how K14 leader cells arise at the leading edge of invading collective clusters of breast tumors, whether localization of these leader cells are essential to direct collective migration, and how leader cells respond to microenvironmental cues in order to direct collective migration. To do so, we combined heterogeneous, primary mouse tumor organoids and microfluidic technology in order to recapitulate multiple in vivo cues of the dynamically changing tumor microenvironment and create a physiologically relevant 3D in vitro model of collective tumor cell migration. Using our platform, we successfully resolve competing hypotheses of leader cell development. Our study demonstrates that a sub-set of pre-existing K14+ cells must spatially reorganize to the leading edge of the tumor to guide collective migration and this requires both CXCR4 and DDR2 signaling.

Furthermore, we reveal that leader cells utilize multiple cues in the tumor microenvironment, including low oxygen, collagen density, chemokine gradient, and interstitial fluid flow, in order to decide how to polarize within a tumor orgnoiad and guide collective migration.

One of the advantages of using a microfluidic model system is the ability to control and mimic multiple features of the tumor microenvironment, which can't be investigated using current in vivo or 2D in vitro methods. For example, interstitial flow transports a chemokine such as SDF1 through the extracellular matrix, thus dramatically altering the spatial distribution [28]. As a result, interstitial fluid flow and the spatial distribution of a chemokine are closely linked. Our platform can control both of these microenvironmental cues. Additionally, we use primary tumor organoids with all their inherent heterogeneity, which preserves in vivo cell-cell and cell-martrix interactions. Prior studies using microfluidic devices have focused on forming 3D aggregates of homogeneous tumor cell lines or mixtures of cell lines [38, 39], and thus do not account for the dynamic and differential response of an inherently heterogeneous cell population.

Our model system displays collective cell migration of tumor cell clusters that is different from previously reported in vitro models which observed collective migration of multicellular tumor cell strands $[3,9,40]$. A recent study of pancreatic, breast, and colon cancer observed clustered collective migration in vivo that differs from the classically described in vitro systems of collective migration of multi-cellular strands with spindle-like protrusions [41]. Findings demonstrate cells have different cellular plasticity that result in different subtypes of collective migration [41]. In vivo studies of breast cancer indicate luminal A and B subtypes of breast cancer, which have a unique cellular plasticity, have a tendency to migrate as clusters compared to other breast cancer subtypes. Together, these prior studies, along with our current study, further emphasizes that a spectrum of collective 
migration behaviors exist, and this may be due to the fact that tumor cells exist in varying states of cellular plasticity.

The development of leader cells, their location within a collectively migrating unit, and their role in collective migration is under debate $[42,43]$. Our study reveals another model of leader cell development: not all cells within the collective unit begin with similar phenotypic features; in fact, only a subset of cells have the capability to be leader cells as evidenced by the real-time migration of K14 positive cells within the organoid and K14-GFP expression maintenance throughout our live-imaging studies. One potential reason our observations are different from prior models of leader cell development could be the presence of dynamic changes in ECM cues. These findings help begin to understand the intricate relationship between microenvironmental cues and leader cell function in initiating, guiding, and maintaining collective migration.

Another key finding from our work is a potential interaction between CXCR4 and DDR2 that contribute to K14 leader cell polarization and collective migration. For MMTV-PyMT breast tumor organoids, we observed both $\mathrm{K} 14+$ and CXCR4+ cell polarization in response to a SDF1 chemokine gradient. When we inhibited CXCR4 or pharmacologically inhibited or genetically deleted Ddr2 these organoids lost their ability to directionally migrate, K14 leader cells did not polarize to a leading edge, and CXCR4 expressing cells no longer localized to the leading edge.

To our knowledge this is the first reported interaction between CXCR4 and DDR2. These effects could be the result of a common or shared signaling component between the two receptors. Prior studies have separately described that both CXCR4 [44] and DDR2 [45] can independently impact integrin $\beta 1$ activity, another collagen binding receptor that regulates metastasis [46]. Another possibility is that CXCR4 signaling affects DDR2 function or vice versa. Since we performed global inhibition or knockout (affect all cell types within the tumor organoid), CXCR4 and DDR2 may have essential, but independent signaling mechnanisms within different cell types of the heterogeneous tumor cluster. In the past, it was believed that cells capable of metastasis expressed a set of genes that provided their ability to disseminate; however, single cell sequencing studies have revealed that circulating tumor cells are also heterogeneous, and the different cell subtypes may have different roles in the process of metastasis [47, 48]. The relationships and potential crosstalk between CXCR4 and DDR2 signals in dictating K14 leader cell polarization and collective migration warrants further investigation.

In our study, we observe a matrix environment that promotes both K14 leader cell polarization and directed collective migration. Some of the matrix features we quantified were stiffness, fiber diameter, and fiber orientation. However, this is not an exhaustive list of matrix features that can contribute to collective migration. Other studies have investigated the independent contributions of ligand density, presentation, and matrix stiffness as well as their synergistic effects on cell migration [49-53]. Based on our current microfluidic device setup, we are unable to decouple the effects of ligand density and matrix stiffness, without simultaneously altering ligand presentation and subsequent cell-matrix interactions that are essential for collective migration. However, in future work, we can alter the design of the 
device such that we can independently control ligand density without impacting matrix stiffness to further investigate influences on K14 polarization and collective migration."

Finally, our study demonstrates that collective migration is sensitive to both the mean concentration of SDF1 as well as the magnitude of the gradient. An interesting result is that the directed migration of tumor organoids in two conditions (groups 37.5_12.5 and 75_25) only occurred during the initial six hours. This may be due to changes in SDF1 concentration and gradient as organoids migrate to different regions of the microfluidic device [54, 55]. Since our microfluidic system is dynamic, as organoids migrate within the device there can be changes in the mean concentration and gradient magnitudes of SDF1. In the current setup, we are unable to delineate the exact location of the organoid within the device (i.e., within the gradient) as our imaging studies require high magnification that does not allow for capture of the entire microfluidic device, thereby limiting the ability to identify where within the gradient the organoids are located.

Before this study, how K14 leader cells arise and respond to microenvironmental cues to lead collective migration, and the effects of CXCR4 and DDR2 in K14 leader cell development were largely unknown. Our study paves the way for future investigations of leader cell driven collective migration and development of therapies that can target leader cell polarization as a means to treat or prevent metastasis.

\section{Supplementary Material}

Refer to Web version on PubMed Central for supplementary material.

\section{Acknowledgements:}

G.D. Longmore received NIH R01CA196205 and U54 CA210173. S.C. George received start-up funds from the School of Engineering at Washington University in St. Louis. P.Y. Hwang received an American Cancer Society Postdoctoral Fellowship 131342-PF-17-238-01-CSM and a WM Keck Foundation Postdoctoral Fellowship. The authors would like to thank the Washington University Center for Cellular Imaging (WUCCI) for microscopy help. The authors would also like to thank Dr. Drew Elizabeth Glaser, Dr. Mary-Kathryn Sewell-Loftin, Dr. Venktesh Shirure, and Benjamin Aunins for their help and advice.

Disclosures: The Longmore laboratory currently receives funding from Pfizer, Inc.

None of the work presented herein was supported by these funds, however.

\section{References}

1. van Helvert S, Storm C, and Friedl P, Mechanoreciprocity in cell migration. Nat Cell Biol, 2018 20(1): p. 8-20. DOI: 10.1038/s41556-017-0012-0. [PubMed: 29269951]

2. Malik R, Lelkes PI, and Cukierman E, Biomechanical and biochemical remodeling of stromal extracellular matrix in cancer. Trends Biotechnol, 2015 33(4): p. 230-6. DOI: 10.1016/j.tibtech. 2015.01.004. [PubMed: 25708906]

3. Cheung KJ, Padmanaban V, Silvestri V, Schipper K, Cohen JD, Fairchild AN, Gorin MA, Verdone JE, Pienta KJ, Bader JS, and Ewald AJ, Polyclonal breast cancer metastases arise from collective dissemination of keratin 14-expressing tumor cell clusters. Proc Natl Acad Sci U S A, 2016 113(7): p. E854-63. DOI: 10.1073/pnas.1508541113. [PubMed: 26831077]

4. Cheung KJ and Ewald AJ, A collective route to metastasis: Seeding by tumor cell clusters. Science, 2016 352(6282): p. 167-9. DOI: 10.1126/science.aaf6546 [PubMed: 27124449] 
5. Stingl J and Caldas C, Molecular heterogeneity of breast carcinomas and the cancer stem cell hypothesis. Nat Rev Cancer, 2007 7(10): p. 791-799 [PubMed: 17851544]

6. Yuan Y, Failmezger H, Rueda OM, Ali HR, Gräf S, Chin S-F, Schwarz RF, Curtis C, Dunning MJ, Bardwell H, Johnson N, Doyle S, Turashvili G, Provenzano E, Aparicio S, Caldas C, and Markowetz F, Quantitative Image Analysis of Cellular Heterogeneity in Breast Tumors Complements Genomic Profiling. Science Translational Medicine, 2012 4(157): p. 157ra143157ra143. DOI: 10.1126/scitranslmed.3004330

7. Friedl P, Locker J, Sahai E, and Segall JE, Classifying collective cancer cell invasion. Nature Cell Biology, 2012 14: p. 777 DOI: 10.1038/ncb2548 [PubMed: 22854810]

8. Camley BA and Rappel WJ, Physical models of collective cell motility: from cell to tissue. J Phys D Appl Phys, 2017 50(11). DOI: 10.1088/1361-6463/aa56fe.

9. Ewald AJ, Brenot A, Duong M, Chan BS, and Werb Z, Collective epithelial migration and cell rearrangements drive mammary branching morphogenesis. Dev Cell, 2008 14(4): p. 570-81. DOI: 10.1016/j.devcel.2008.03.003. [PubMed: 18410732]

10. Cheung Kevin J., Gabrielson E, Werb Z, and Ewald Andrew J., Collective Invasion in Breast Cancer Requires a Conserved Basal Epithelial Program. Cell. 155(7): p. 1639-1651. DOI: 10.1016/j.cell.2013.11.029

11. Nguyen-Ngoc K-V, Cheung KJ, Brenot A, Shamir ER, Gray RS, Hines WC, Yaswen P, Werb Z, and Ewald AJ, ECM microenvironment regulates collective migration and local dissemination in normal and malignant mammary epithelium. Proceedings of the National Academy of Sciences, 2012 109(39): p. E2595-E2604. DOI: 10.1073/pnas.1212834109

12. Stuelten CH, Parent CA, and Montell DJ, Cell motility in cancer invasion and metastasis: insights from simple model organisms. Nat Rev Cancer, 2018 DOI: 10.1038/nrc.2018.15

13. Ruprecht V, Monzo P, Ravasio A, Yue Z, Makhija E, Strale PO, Gauthier N, Shivashankar GV, Studer V, Albiges-Rizo C, and Viasnoff V, How cells respond to environmental cues - insights from bio-functionalized substrates. Journal of Cell Science, 2017 130(1): p. 51-61. DOI: 10.1242/ jcs.196162 [PubMed: 27856508]

14. Brantley-Sieders DM, Fang WB, Hicks DJ, Zhuang G, Shyr Y, and Chen J, Impaired tumor microenvironment in EphA2-deficient mice inhibits tumor angiogenesis and metastatic progression. FASEB J, 2005 19(13): p. 1884-6. DOI: 05-4038fje [pii] 10.1096/fj.05-4038fje [PubMed: 16166198]

15. Zhang Z, Ramirez NE, Yankeelov TE, Li Z, Ford LE, Qi Y, Pozzi A, and Zutter MM, alpha2beta1 integrin expression in the tumor microenvironment enhances tumor angiogenesis in a tumor cellspecific manner. Blood, 2008 111(4): p. 1980-8. DOI: blood-2007-06-094680 [pii] 10.1182/ blood-2007-06-094680. [PubMed: 18042800]

16. Lin B, Yin T, Wu YI, Inoue T, and Levchenko A, Interplay between chemotaxis and contact inhibition of locomotion determines exploratory cell migration. Nat Commun, 2015 6: p. 6619 DOI: 10.1038/ncomms7619. [PubMed: 25851023]

17. Spill F, Reynolds DS, Kamm RD, and Zaman MH, Impact of the physical microenvironment on tumor progression and metastasis. Current Opinion in Biotechnology, 2016 40: p. 41-48. DOI: 10.1016/j.copbio.2016.02.007 [PubMed: 26938687]

18. Munson JM, Bellamkonda RV, and Swartz MA, Interstitial flow in a 3D microenvironment increases glioma invasion by a CXCR4-dependent mechanism. Cancer Res, 2013 73(5): p. 153646. DOI: 10.1158/0008-5472.can-12-2838 [PubMed: 23271726]

19. Zhang C, Fu L, Fu J, Hu L, Yang H, Rong TH, Li Y, Liu H, Fu SB, Zeng YX, and Guan XY, Fibroblast growth factor receptor 2-positive fibroblasts provide a suitable microenvironment for tumor development and progression in esophageal carcinoma. Clin Cancer Res, 2009 15(12): p. 4017-27. DOI: 1078-0432.CCR-08-2824 [pii] 10.1158/1078-0432.CCR-08-2824 [PubMed: 19509166]

20. Fuchigami T, Koyama H, Kishida M, Nishizawa Y, Iijima M, Kibe T, Ueda M, Kiyono T, Maniwa Y, Nakamura N, and Kishida S, Fibroblasts promote the collective invasion of ameloblastoma tumor cells in a 3D coculture model. FEBS Open Bio, 2017 7(12): p. 2000-2007. DOI: $10.1002 / 2211-5463.12313$ 
21. Kalli M and Stylianopoulos T, Defining the Role of Solid Stress and Matrix Stiffness in Cancer Cell Proliferation and Metastasis. Frontiers in Oncology, 2018 8(55). DOI: 10.3389/fonc. 2018.00055

22. Kim MC, Abeyaratne R, Kamm RD, and Asada HH. Dynamic modeling of cancer cell migration in an extracellular matrix fiber network. in 2017 American Control Conference (ACC). 2017.

23. Wang T, Hamilla S, Cam M, Aranda-Espinoza H, and Mili S, Extracellular matrix stiffness and cell contractility control RNA localization to promote cell migration. Nature Communications, 2017 8(1): p. 896 DOI: 10.1038/s41467-017-00884-y

24. Gurski LA, Jha AK, Zhang C, Jia X, and Farach-Carson MC, Hyaluronic acid-based hydrogels as 3D matrices for in vitro evaluation of chemotherapeutic drugs using poorly adherent prostate cancer cells. Biomaterials, 2009 30(30): p. 6076-85. DOI: S0142-9612(09)00786-8 [pii] 10.1016/ j.biomaterials.2009.07.054.2782556. [PubMed: 19695694]

25. Serebriiskii I, Castello-Cros R, Lamb A, Golemis EA, and Cukierman E, Fibroblast-derived 3D matrix differentially regulates the growth and drug-responsiveness of human cancer cells. Matrix Biol, 2008 27(6): p. 573-85. DOI: S0945-053X(08)00039-5 [pii] 10.1016/j.matbio. 2008.02.008.2603546. [PubMed: 18411046]

26. Weigelt B, Lo AT, Park CC, Gray JW, and Bissell MJ, HER2 signaling pathway activation and response of breast cancer cells to HER2-targeting agents is dependent strongly on the 3D microenvironment. Breast Cancer Res Treat, 2010 122(1): p. 35-43. DOI: 10.1007/ s10549-009-0502-2.2935800. [PubMed: 19701706]

27. Marlow R, Honeth G, Lombardi S, Cariati M, Hessey S, Pipili A, Mariotti V, Buchupalli B, Foster K, Bonnet D, Grigoriadis A, Rameshwar P, Purushotham A, Tutt A, and Dontu G, A novel model of dormancy for bone metastatic breast cancer cells. Cancer Res, 2013 73(23): p. 6886-99. DOI: 10.1158/0008-5472.CAN-13-0991 [PubMed: 24145351]

28. Shirure VS, Lezia A, Tao A, Alonzo LF, and George SC, Low levels of physiological interstitial flow eliminate morphogen gradients and guide angiogenesis. Angiogenesis, 2017 DOI: 10.1007/ s10456-017-9559-4

29. Vaezi A, Bauer C, Vasioukhin V, and Fuchs E, Actin cable dynamics and Rho/Rock orchestrate a polarized cytoskeletal architecture in the early steps of assembling a stratified epithelium. Dev Cell, 2002 3(3): p. 367-81 [PubMed: 12361600]

30. Corsa CA, Brenot A, Ponik SM, Liu Y, Zhang K, Van Hove S, Eliceiri KW, Keely PJ, Longmore GD, Loza A, Grither W, and Denardo D, The action of Discoidin Domain Receptor 2 in basal breast tumor cells and tumor stromal Cancer Associated Fibroblasts is critical for breast cancer metastasis. Cell Rep, 20166 14;15(11):2510-23. doi: 10.1016/j.celrep.2016.05.033. Epub 2016 Jun 2 [PubMed: 27264173]

31. Grither WR and Longmore GD, Inhibition of tumor-microenvironment interaction and tumor invasion by small-molecule allosteric inhibitor of DDR2 extracellular domain. Proc Natl Acad Sci U S A, 2018 115(33): p. E7786-e7794. DOI: 10.1073/pnas.1805020115. [PubMed: 30061414]

32. Mayor R and Etienne-Manneville S, The front and rear of collective cell migration. Nat Rev Mol Cell Biol, 2016 17(2): p. 97-109. DOI: 10.1038/nrm.2015.14 [PubMed: 26726037]

33. Li H, Xu F, Li S, Zhong A, Meng X, and Lai M, The tumor microenvironment: An irreplaceable element of tumor budding and epithelial-mesenchymal transition-mediated cancer metastasis. Cell Adh Migr, 2016 10(4): p. 434-46. DOI: 10.1080/19336918.2015.1129481. [PubMed: 26743180]

34. Ren T, Zhang W, Liu X, Zhao H, Zhang J, Zhang J, Li X, Zhang Y, Bu X, Shi M, Yao L, and Su J, Discoidin domain receptor 2 (DDR2) promotes breast cancer cell metastasis and the mechanism implicates epithelial-mesenchymal transition programme under hypoxia. J Pathol, 2014 234(4): p. 526-37. DOI: 10.1002/path.4415 [PubMed: 25130389]

35. Cronin PA, Wang JH, and Redmond HP, Hypoxia increases the metastatic ability of breast cancer cells via upregulation of CXCR4. BMC Cancer, 2010 10: p. 225 DOI: 10.1186/1471-2407-10-225. [PubMed: 20492653]

36. Gilkes DM and Semenza GL, Role of hypoxia-inducible factors in breast cancer metastasis. Future Oncol, 2013 9(11): p. 1623-36. DOI: 10.2217/fon.13.92. [PubMed: 24156323]

37. Li S, Shen D, Shao J, Crowder R, Liu W, Prat A, He X, Liu S, Hoog J, Lu C, Ding L, Griffith OL, Miller C, Larson D, Fulton RS, Harrison M, Mooney T, McMichael JF, Luo J, Tao Y, Goncalves R, 
Schlosberg C, Hiken JF, Saied L, Sanchez C, Giuntoli T, Bumb C, Cooper C, Kitchens RT, Lin A, Phommaly C, Davies SR, Zhang J, Kavuri MS, McEachern D, Dong YY, Ma C, Pluard T, Naughton M, Bose R, Suresh R, McDowell R, Michel L, Aft R, Gillanders W, DeSchryver K, Wilson RK, Wang S, Mills GB, Gonzalez-Angulo A, Edwards JR, Maher C, Perou CM, Mardis $\mathrm{ER}$, and Ellis MJ, Endocrine-therapy-resistant ESR1 variants revealed by genomic characterization of breast-cancer-derived xenografts. Cell Rep, 2013 4(6): p. 1116-30. DOI: 10.1016/j.celrep. 2013.08.022. [PubMed: 24055055]

38. Piotrowski-Daspit AS, Tien J, and Nelson CM, Interstitial fluid pressure regulates collective invasion in engineered human breast tumors via Snail, vimentin, and E-cadherin. Integr Biol (Camb), 2016 8(3): p. 319-31. DOI: 10.1039/c5ib00282f. [PubMed: 26853861]

39. Aizel K, Clark AG, Simon A, Geraldo S, Funfak A, Vargas P, Bibette J, Vignjevic DM, and Bremond N, A tuneable microfluidic system for long duration chemotaxis experiments in a $3 \mathrm{D}$ collagen matrix. Lab Chip, 2017 17(22): p. 3851-3861. DOI: 10.1039/c7lc00649g [PubMed: 29022983]

40. Clark AG and Vignjevic DM, Modes of cancer cell invasion and the role of the microenvironment. Current Opinion in Cell Biology, 2015 36: p. 13-22. DOI: 10.1016/j.ceb.2015.06.004 [PubMed: 26183445]

41. Aiello NM, Maddipati R, Norgard RJ, Balli D, Li J, Yuan S, Yamazoe T, Black T, Sahmoud A, Furth EE, Bar-Sagi D, and Stanger BZ, EMT Subtype Influences Epithelial Plasticity and Mode of Cell Migration. Dev Cell, 2018 45(6): p. 681-695.e4 DOI: 10.1016/j.devcel.2018.05.027. [PubMed: 29920274]

42. Uechi H and Kuranaga E, Mechanisms of collective cell movement lacking a leading or free front edge in vivo. Cellular and Molecular Life Sciences, 2017 74(15): p. 2709-2722. DOI: 10.1007/ s00018-017-2489-x [PubMed: 28243700]

43. Hallou A, Jennings J, and Kabla AJ, Tumour heterogeneity promotes collective invasion and cancer metastatic dissemination. Royal Society Open Science, 2017 4(8). DOI: 10.1098/rsos. 161007

44. Izumi D, Ishimoto T, Miyake K, Sugihara H, Eto K, Sawayama H, Yasuda T, Kiyozumi Y, Kaida T, Kurashige J, Imamura Y, Hiyoshi Y, Iwatsuki M, Iwagami S, Baba Y, Sakamoto Y, Miyamoto Y, Yoshida N, Watanabe M, Takamori H, Araki N, Tan P, and Baba H, CXCL12/CXCR4 activation by cancer-associated fibroblasts promotes integrin $\beta 1$ clustering and invasiveness in gastric cancer. International Journal of Cancer, 2016 138(5): p. 1207-1219. DOI: 10.1002/ijc.29864 [PubMed: 26414794]

45. Petty JM, Lenox CC, Weiss DJ, Poynter ME, and Suratt BT, Crosstalk between CXCR4/stromal derived factor-1 and VLA-4/VCAM-1 pathways regulates neutrophil retention in the bone marrow. J Immunol, 2009 182(1): p. 604-12. [PubMed: 19109194]

46. Pan B, Guo J, Liao Q, and Zhao Y, beta1 and beta3 integrins in breast, prostate and pancreatic cancer: A novel implication. Oncol Lett, 2018 15(4): p. 5412-5416. DOI: 10.3892/ol.2018.8076. [PubMed: 29556293]

47. Riggi N, Aguet M, and Stamenkovic I, Cancer Metastasis: A Reappraisal of Its Underlying Mechanisms and Their Relevance to Treatment. Annu Rev Pathol, 2018 13: p. 117-140. DOI: 10.1146/annurev-pathol-020117-044127 [PubMed: 29068753]

48. Puram SV, Tirosh I, Parikh AS, Patel AP, Yizhak K, Gillespie S, Rodman C, Luo CL, Mroz EA, Emerick KS, Deschler DG, Varvares MA, Mylvaganam R, Rozenblatt-Rosen O, Rocco JW, Faquin WC, Lin DT, Regev A, and Bernstein BE, Single-Cell Transcriptomic Analysis of Primary and Metastatic Tumor Ecosystems in Head and Neck Cancer. Cell, 2017 171(7): p. 1611-1624.e24 DOI: 10.1016/j.cell.2017.10.044 [PubMed: 29198524]

49. Zaman MH, Trapani LM, Sieminski AL, Mackellar D, Gong H, Kamm RD, Wells A, Lauffenburger DA, and Matsudaira P, Migration of tumor cells in 3D matrices is governed by matrix stiffness along with cell-matrix adhesion and proteolysis. Proc Natl Acad Sci U S A, 2006 103(29): p. 10889-94. DOI: 10.1073/pnas.0604460103. [PubMed: 16832052]

50. Discher DE, Janmey P, and Wang YL, Tissue cells feel and respond to the stiffness of their substrate. Science, 2005 310(5751): p. 1139-43. DOI: 10.1126/science.1116995 [PubMed: 16293750] 
51. Ulrich TA, Jain A, Tanner K, MacKay JL, and Kumar S, Probing cellular mechanobiology in threedimensional culture with collagen-agarose matrices. Biomaterials, 2010 31(7): p. 1875-84. DOI: 10.1016/j.biomaterials.2009.10.047 [PubMed: 19926126]

52. Maheshwari G, Brown G, Lauffenburger DA, Wells A, and Griffith LG, Cell adhesion and motility depend on nanoscale RGD clustering. J Cell Sci, 2000113 (Pt 10): p. 1677-86 [PubMed: 10769199]

53. Sheetz MP, Felsenfeld DP, and Galbraith CG, Cell migration: regulation of force on extracellularmatrix-integrin complexes. Trends Cell Biol, 1998 8(2): p. 51-4 [PubMed: 9695809]

54. Neel NF, Schutyser E, Sai J, Fan GH, and Richmond A, Chemokine receptor internalization and intracellular trafficking. Cytokine Growth Factor Rev, 2005 16(6): p. 637-58. DOI: 10.1016/ j.cytogfr.2005.05.008. [PubMed: 15998596]

55. Borroni EM, Mantovani A, Locati M, and Bonecchi R, Chemokine receptors intracellular trafficking. Pharmacology \& Therapeutics, 2010 127(1): p. 1-8. DOI: 10.1016/j.pharmthera. 2010.04.006 [PubMed: 20451553] 


\section{Statement of Significance}

Findings demonstrate that pre-existing, randomly distributed leader cells within primary tumor organoids use CXCR4 and DDR2 to polarize to the leading edge and direct migration. 
A

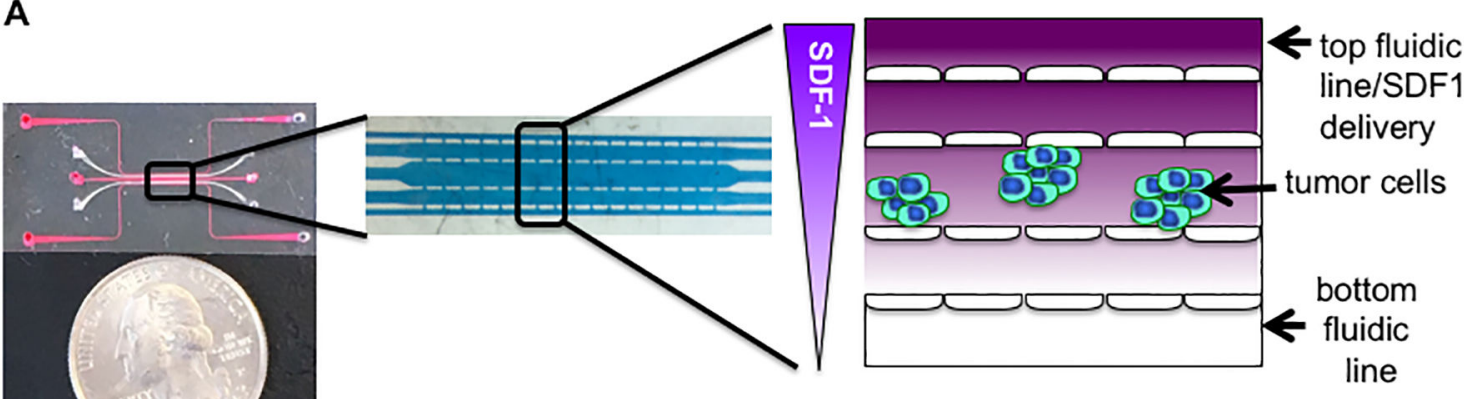

B

C
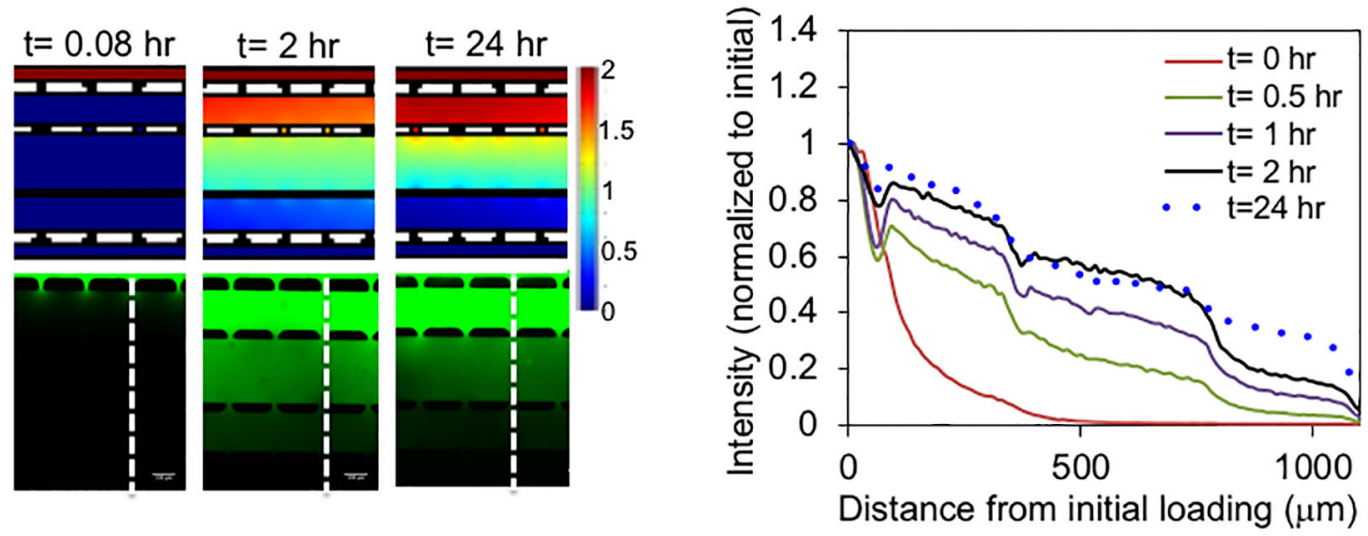

Figure 1: Microfluidic device design and verification

(A) Schematic of microfluidic device design ( 3 tissue chambers flanked by 2 fluidic lines; white ovals are ports seperating the tissue chambers that allow for diffusion of chemokine gradients between them) (B) COMSOL modeling along with experimental verification that we establish an SDF1 chemokine gradient that is maintained for 24 hours (10 kDa FITCDextran used to model SDF1 (8 kDa)) (C) Quantification of FITC-Dextran intensity across the tissue chambers in the device (as identified by the white dotted line in (B)). 


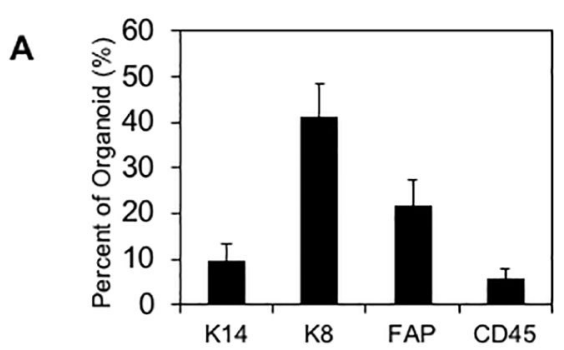

B
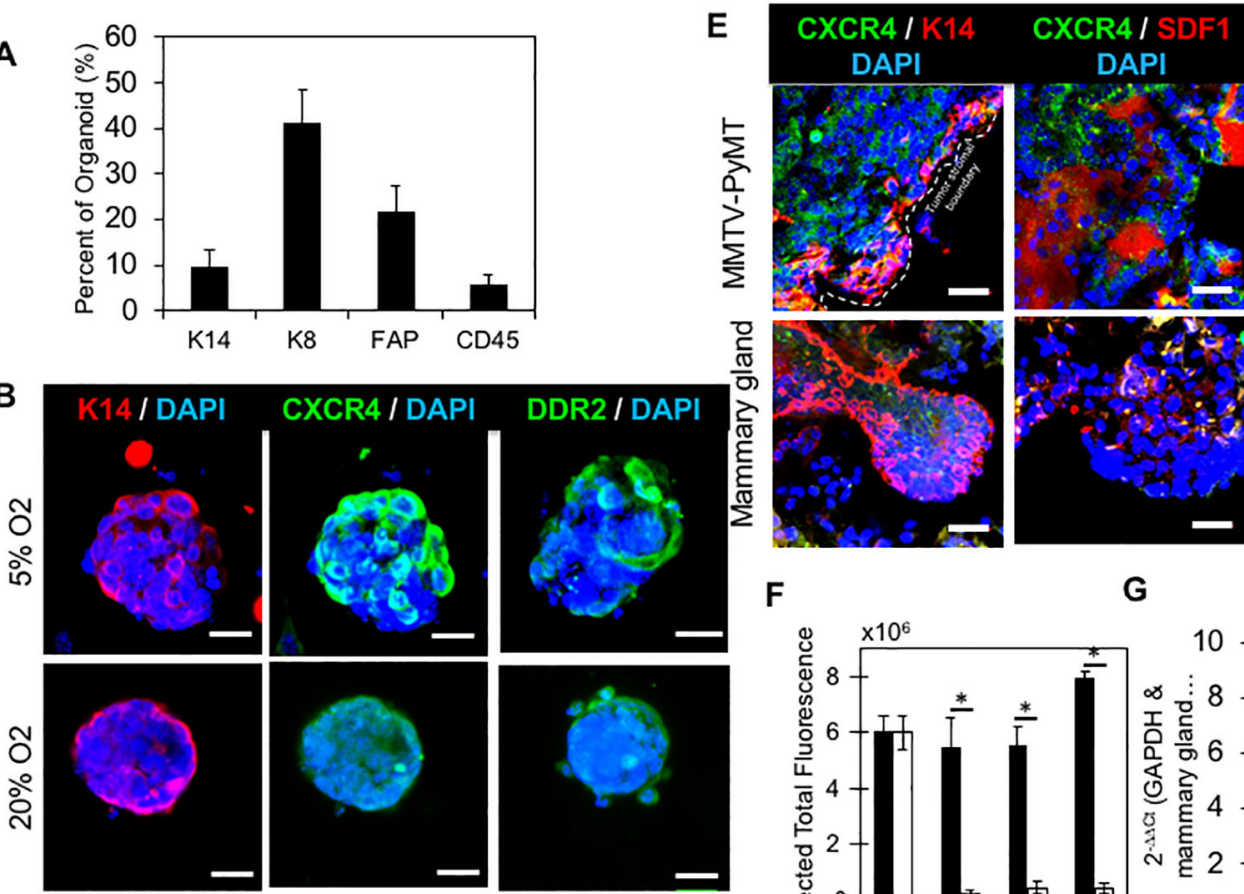

DDR2 / K14
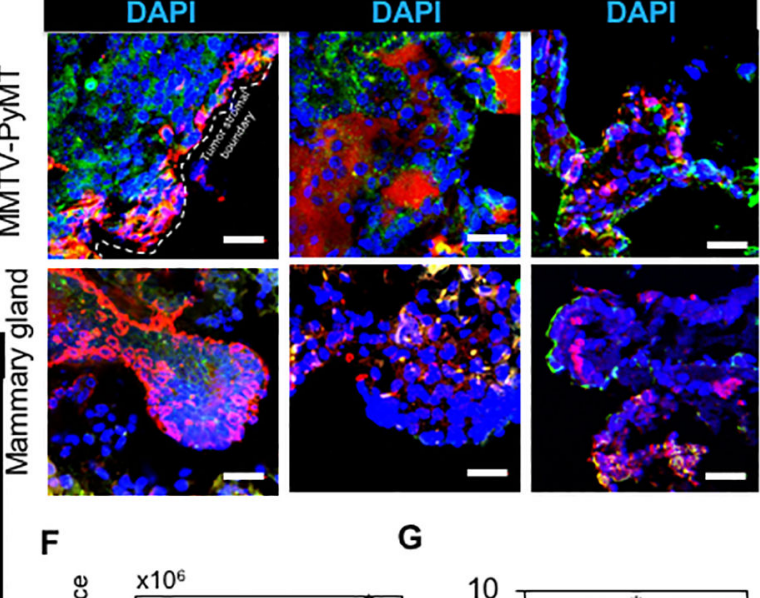

$\mathbf{F}$

G
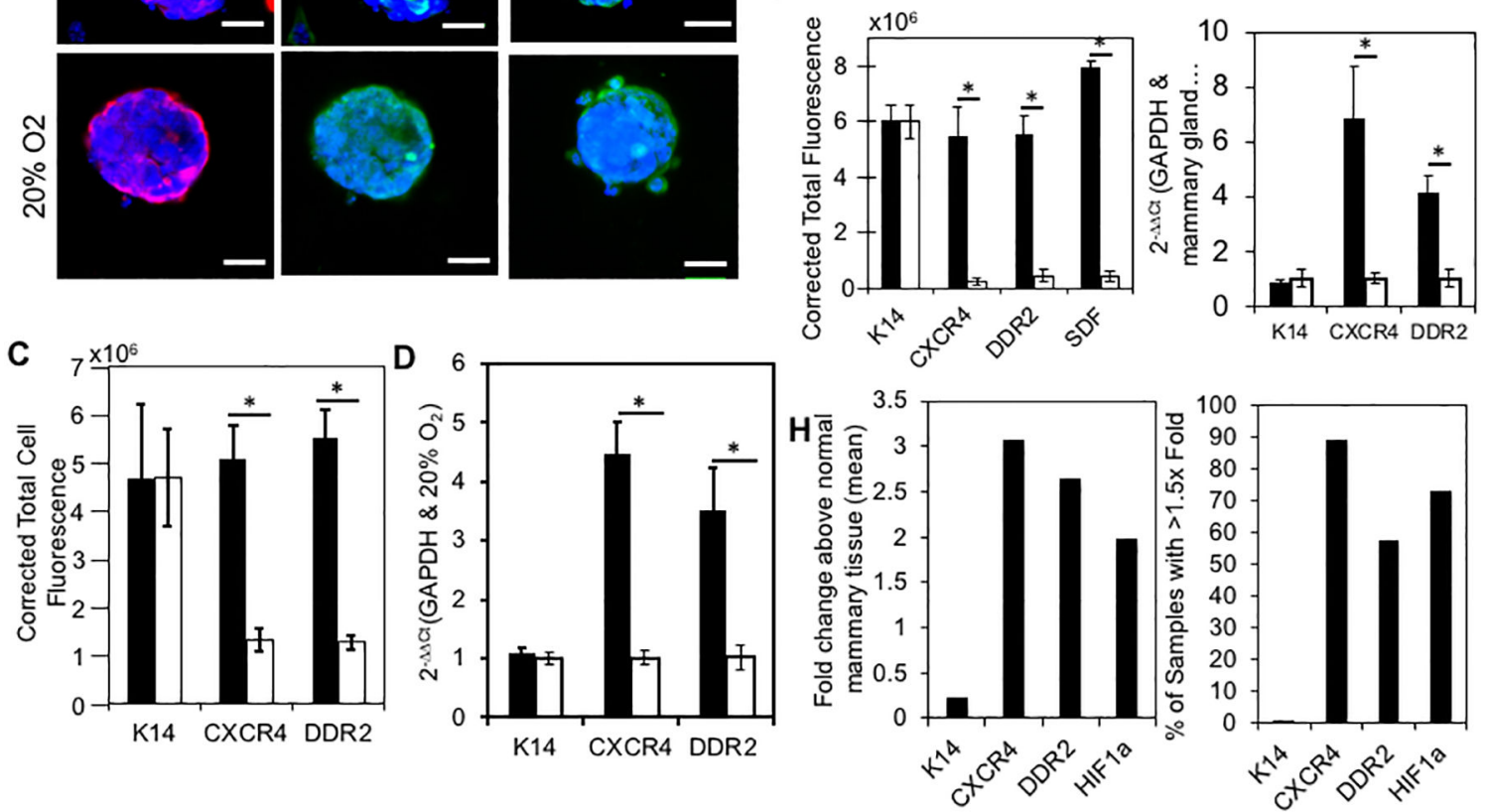

Figure 2: CXCR4 and DDR2 but not K14 expression is upregulated in tumor organoids after exposure to low oxygen as well as breast tumor tissue in vivo

(A) Percent of area for various cell subtypes within heterogeneous tumor organoids: epithelial leader cells (K14), follower epithelial cells (K8), fibroblasts (FAP), and immune (CD45) cells (B) K14 (red), CXCR4 (green), DDR2 (green), and DAPI (blue) expression in mouse tumor organoids $\left(5 \% \mathrm{O}_{2}\right.$ and $\left.20 \% \mathrm{O}_{2}\right)($ scale bar $=25 \mu \mathrm{m})(\mathrm{C})$ Quantification of $\mathrm{K} 14$, CXCR4, and DDR2 expression (black bar: $5 \% \mathrm{O}_{2}$ and white bar: $20 \% \mathrm{O}_{2}$ ) (D) Gene expression (K14, CXCR4, and DDR2) for primary tumor organoids (black bar: 5\% $\mathrm{O}_{2}$ and white bar: $20 \% \mathrm{O}_{2}$ ) (E) K14 (red), CXCR4 (green), SDF1 (red), and DDR2 (green) with DAPI expression in primary MMTV-PyMT breast tissue compared to normal mammary tissue (scale bar $=25 \mu \mathrm{m})(\mathrm{F})$ Quantification of K14, CXCR4, DDR2, and SDF1 expression in primary MMTV-PyMT breast tissue (black bar) compared to normal mmary tissue (white bar) (G) Gene expression (K14, CXCR4, and DDR2) in primary MMTV-PyMT breast tissue (black bar) compared to normal mammary tissue (white bar) $(\mathrm{H})$ Microarray analysis for changes in K14, CXCR4, DDR2, and Hif1a expression in primary human breast tumor 
samples compared to normal mammary gland tissue (for all experiments: *p<0.05; ANOVA with Tukey's post-hoc analysis) 

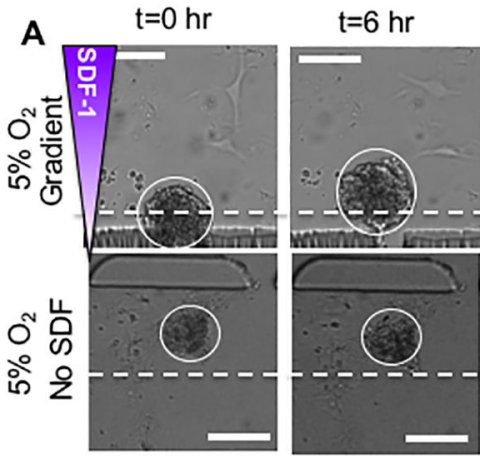

B

$5 \% \mathrm{O}_{2}$ Gradient

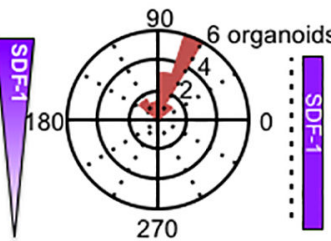

E
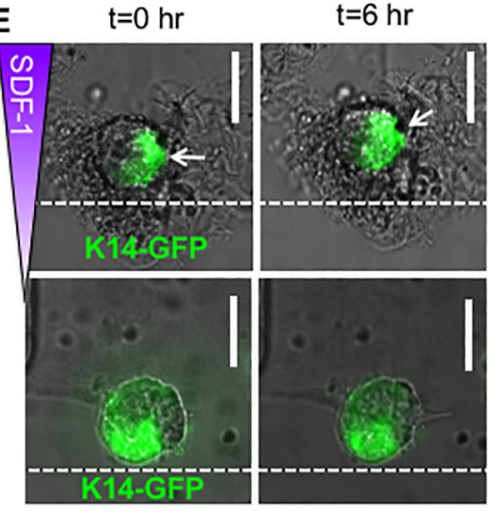

$\mathrm{t}=8 \mathrm{hr}$
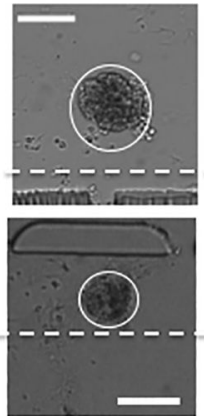

$5 \% \mathrm{O}_{2}$

No Gradient

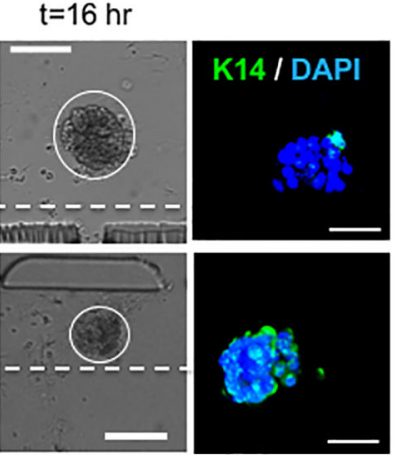

$5 \% \mathrm{O}_{2}$
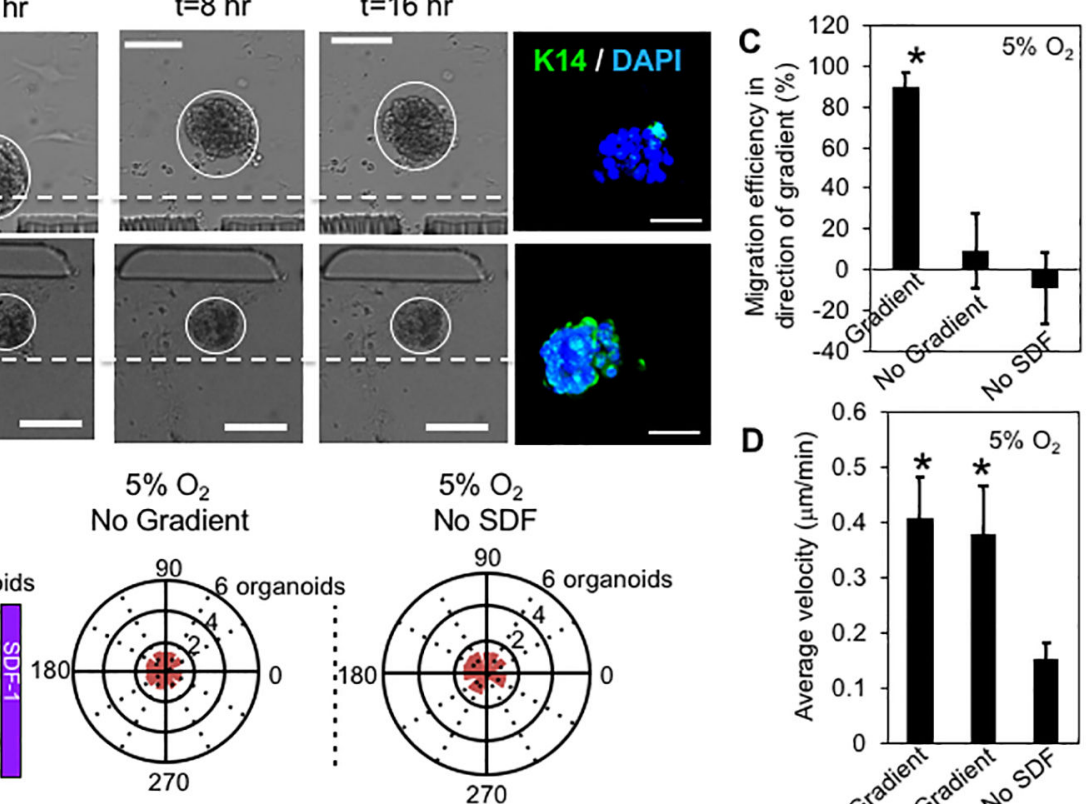

D

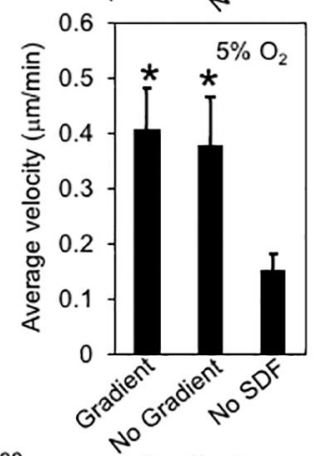

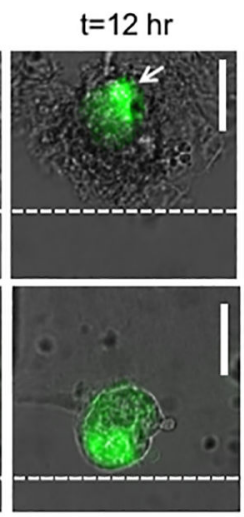
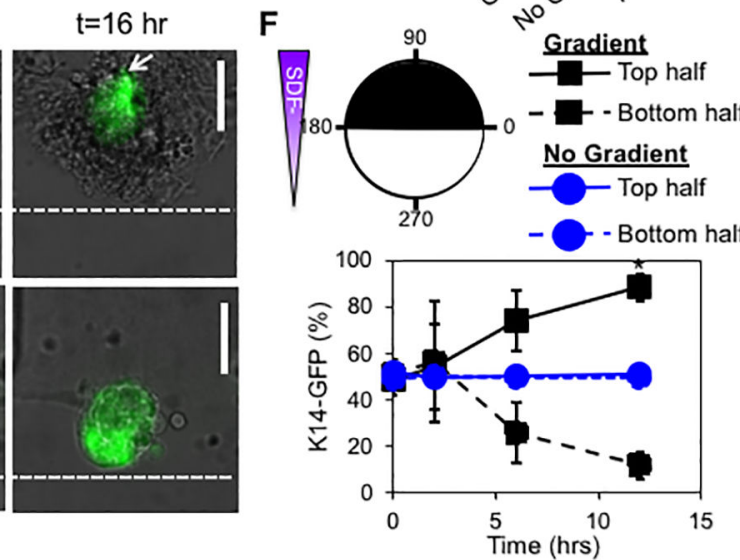

Figure 3: K14 positive cells migrate and polarize within the organoid toward the highest concentration of SDF1 to lead directed collective migration

(A) Time-lapse images of collective migration $\left(5 \% \mathrm{O}_{2}\right)$ with immunostaining of $\mathrm{K} 14$ (green) and DAPI (blue) at the end of experiment (scale bar $=100 \mu \mathrm{m}$ ) (B) Rose plots displaying migration direction $\left(5 \% \mathrm{O}_{2}\right.$; gradient, no gradient, and no SDF conditions) (C-D) Collective migration efficiency and average velocity in the direction of chemokine gradient $\left(5 \% \mathrm{O}_{2}\right)(\mathrm{E})$ Time-lapse images of K14-GFP MMTV-PyMT organoids (scale bar $=50 \mu \mathrm{m})(\mathrm{F}) \mathrm{K} 14-\mathrm{GFP}$ fluorescence over time (for all experiments: *p<0.05, ANOVA with Tukey's post-hoc analysis; gradient conditions $=50 \_0$ ) 

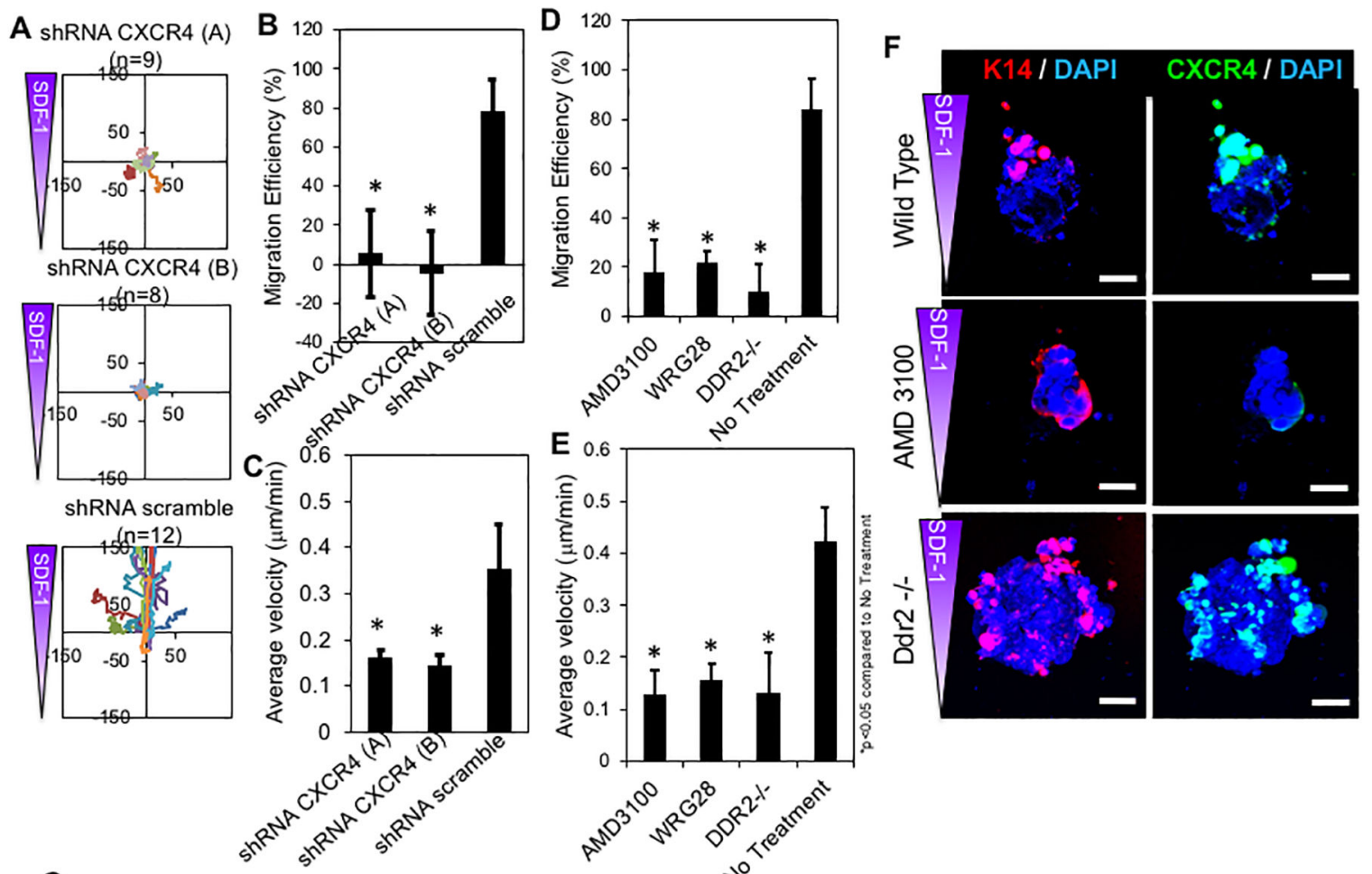

G

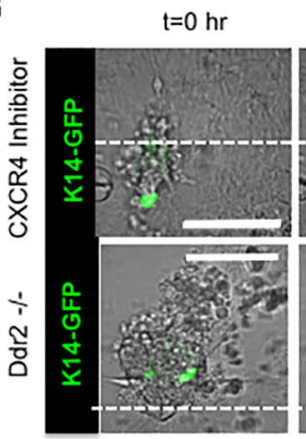

$\mathrm{t}=6 \mathrm{hr}$
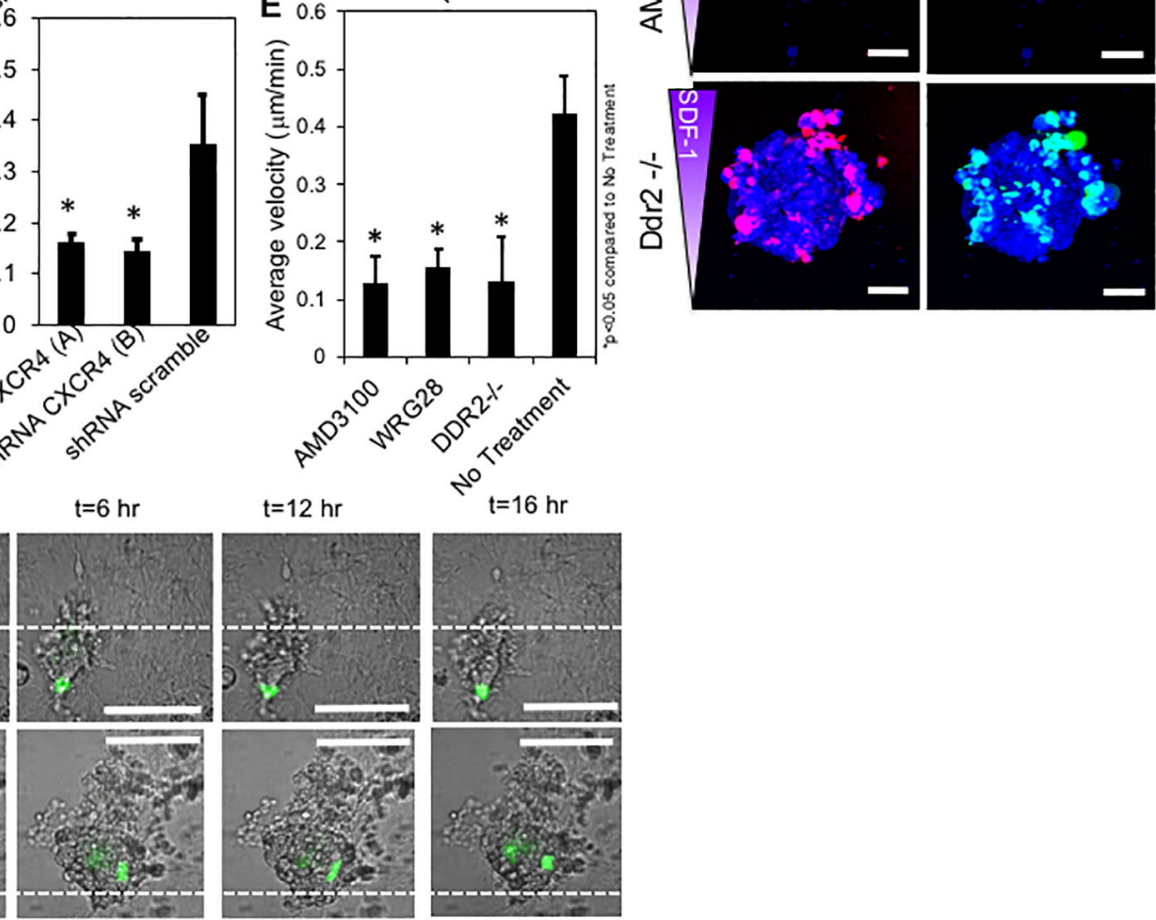

Figure 4: Directed collective migration in tumor organoids is SDF1-responsive via CXCR4 and DDR2

(A) Cell tracking for shRNA-CXCR4 primary organoids (axes $=\mu \mathrm{m}$; two different shRNACXCR4 constructs) (B-C) Collective migration efficiency and average velocity after CXCR4 knockdown (compared to scramble control) (D-E) Collective migration efficiency and average velocity after CXCR4 inhibitor (AMD3100), DDR2 inhibitor (WRG-28), or global DDR2-/- MMTV-PyMT tumor organoids (compared to No Treatment control) (F) K14 (red) and CXCR4 (green) with DAPI (blue) expression for tumor organoids after AMD3100 treatment or global DDR2-l- (scale bar $=25 \mu \mathrm{m})(\mathrm{G})$ Time-lapse images after treatment with CXCR4 inhibitor, AMD3100, or global DDR2-/- (scale bar $=25 \mu \mathrm{m}$ ) (for all experiments: ${ }^{*} \mathrm{p}<0.05$, ANOVA with Tukey's post-hoc analysis; gradient conditions $=50 \_0$ ) 

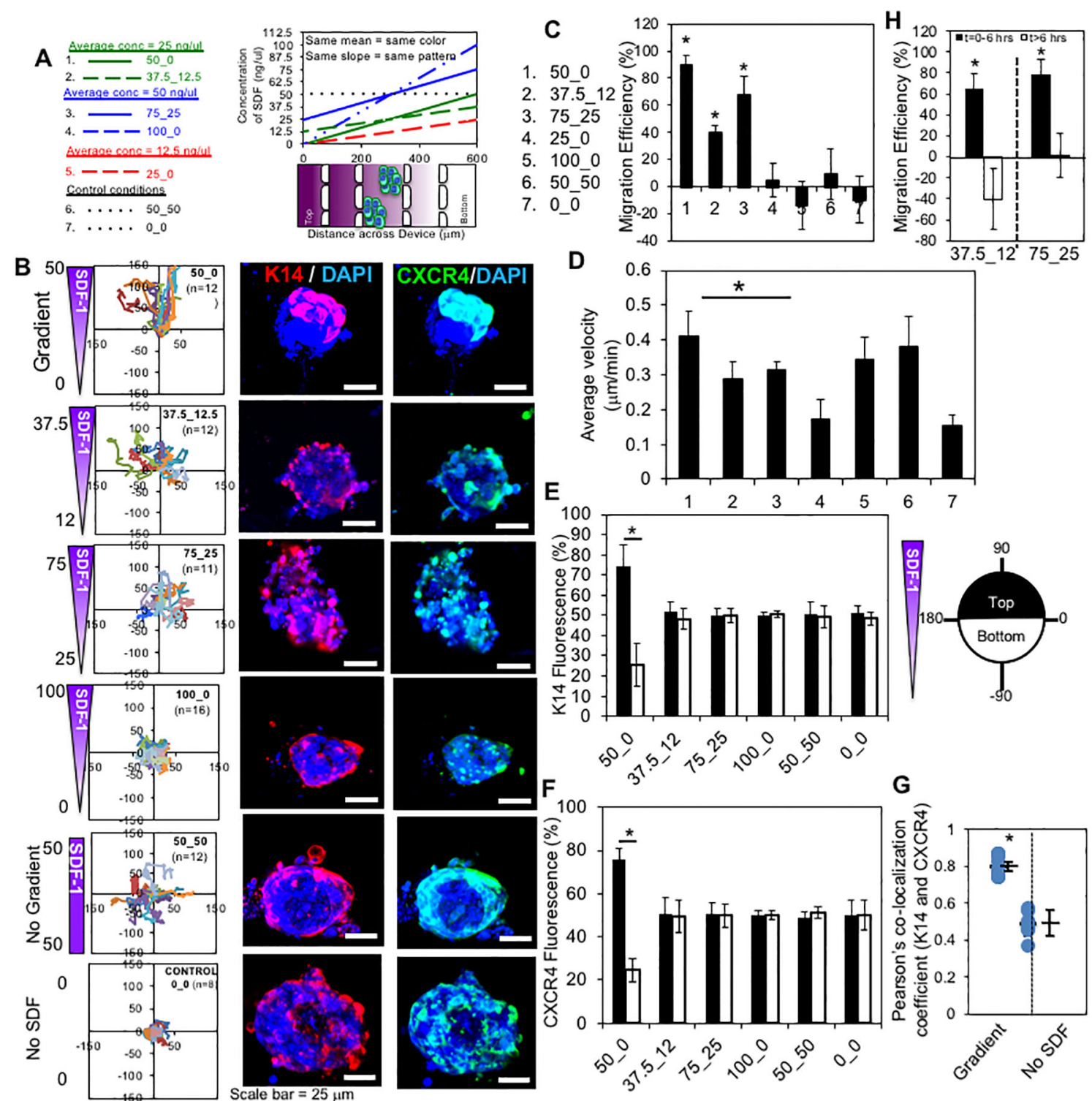

Figure 5: Initiation and direction of collective migration in tumor organoids is dependent on chemokine gradient mean and magnitude

(A) Schematic of the different SDF1 chemokine gradient conditions investigated (B) Cell tracking (axes $=\mu \mathrm{m}$; each line represents 1 collective organoid; $n=8-16$ per group) and immunostaining (K14 (red) and CXCR4 (green) with DAPI (blue)) in response to various gradient shapes and magnitudes (scale bar $=25 \mu \mathrm{m})(\mathrm{C})$ Collective migration efficiency and (D) average velocity (E) K14 and (F)CXCR4 fluorescence in top (higher concentration of SDF1) or bottom half of organoids (G) Pearson's co-localization coefficient analysis for CXCR4 and K14 expression overlap after exposure to chemokine gradient compared to "No SDF1" conditions (blue dots = percent of CXCR4 and K14 staining overlap in each organoid, black bars $=$ average for all samples with standard deviation) $(\mathrm{H})$ Collective migration efficiency for groups 37.5_12.5 and groups 75_25 split into two groups: $\mathrm{t}=0-6$ hours and 6-18hrs (for all experiments: *p<0.05, ANOVA with Tukey's post-hoc analysis) 

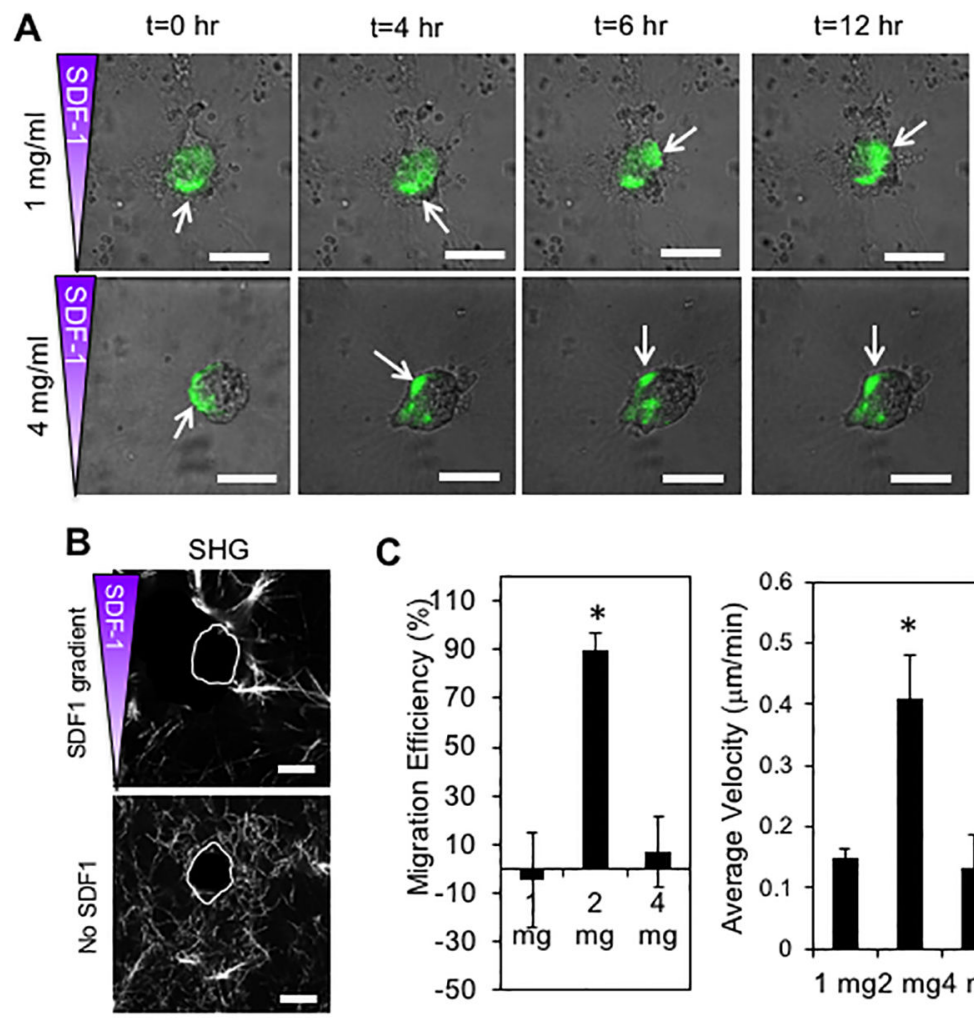

C
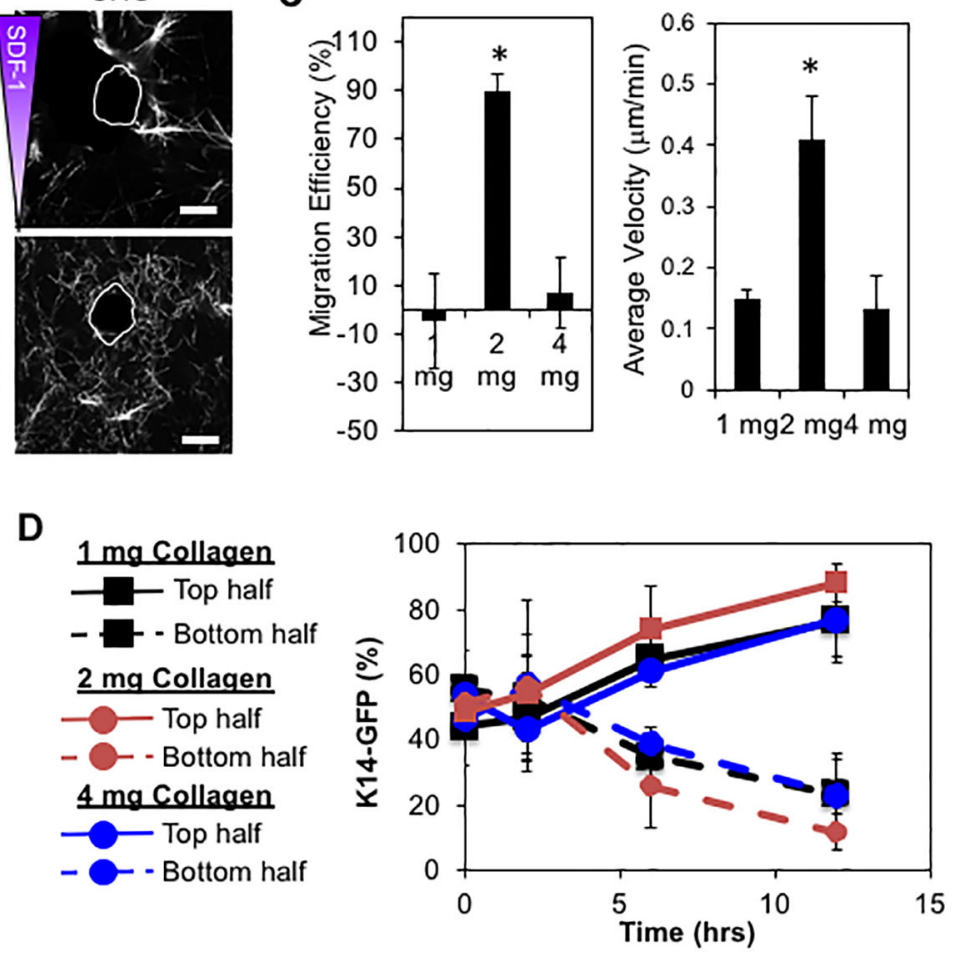

Figure 6: Collagen matrix properties impact collective migration but not K14 polarization (A) Time-lapse images of K14-GFP MMTV-PyMT primary organoids (arrows indicate K14GFP cells; scale bar $=50 \mu \mathrm{m})($ B) SHG imaging of remodeled collagen $($ scale bar $=25 \mu \mathrm{m})$ (C) Collective migration efficiency and average velocity in for various collagen hydrogels (D) K14-GFP fluorescence within tumor organoids (for all experiments: * $\mathrm{p}<0.05$, ANOVA with Tukey's post-hoc analysis; gradient conditions $=50 \_0$ ) 


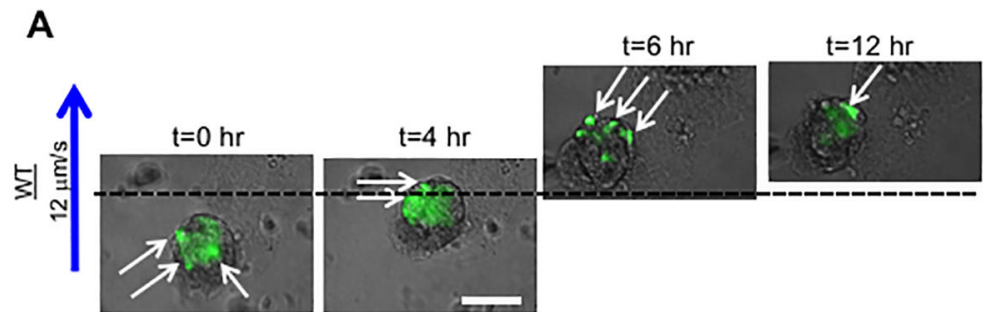

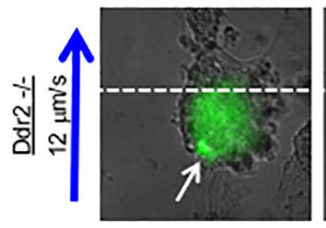

B
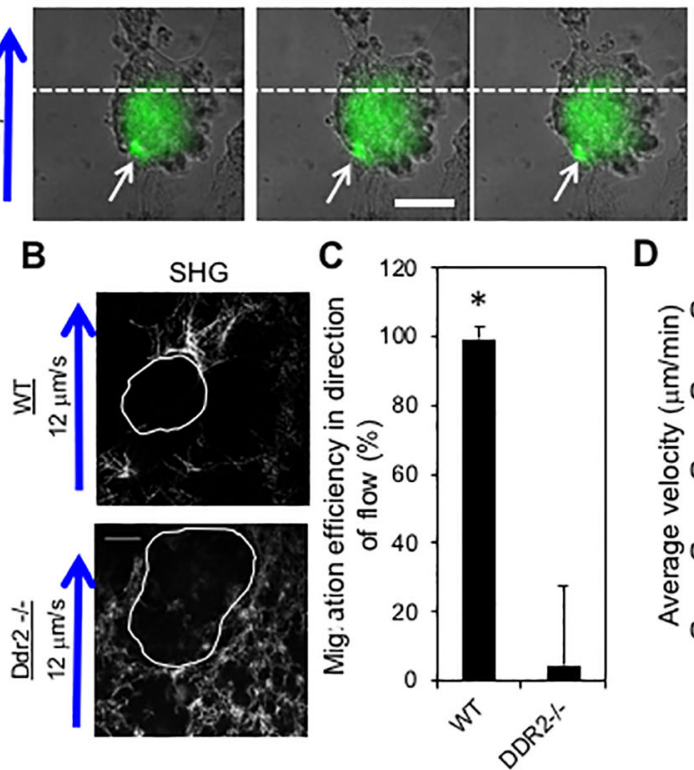

D
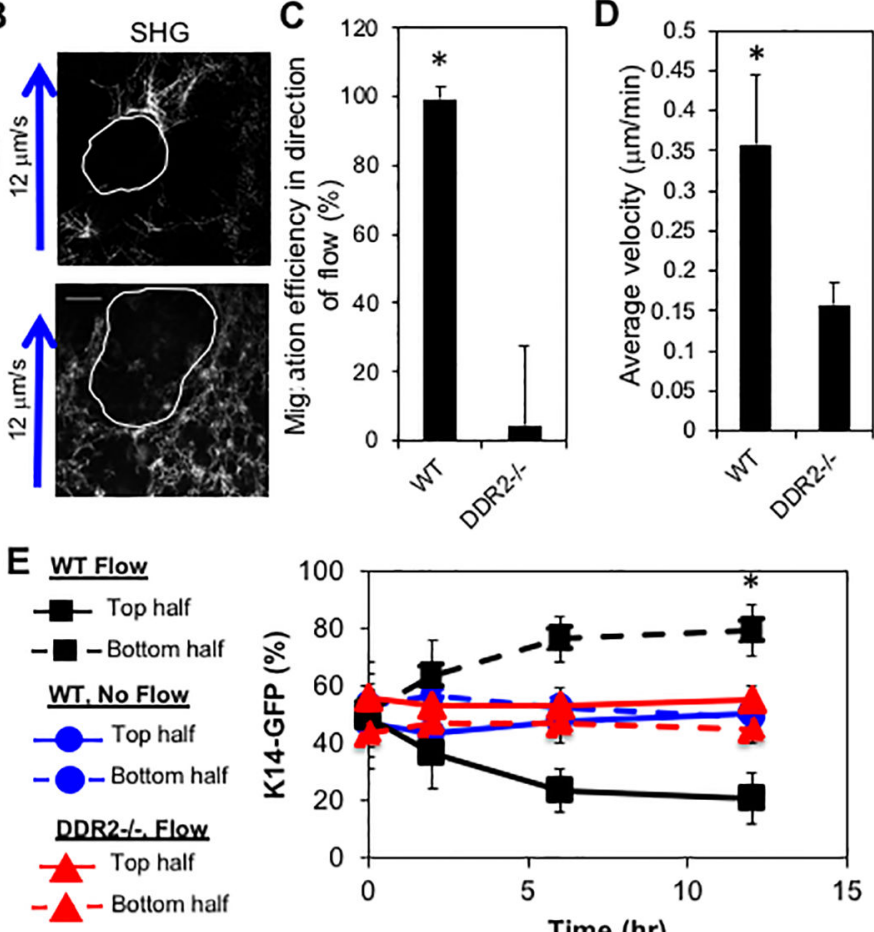

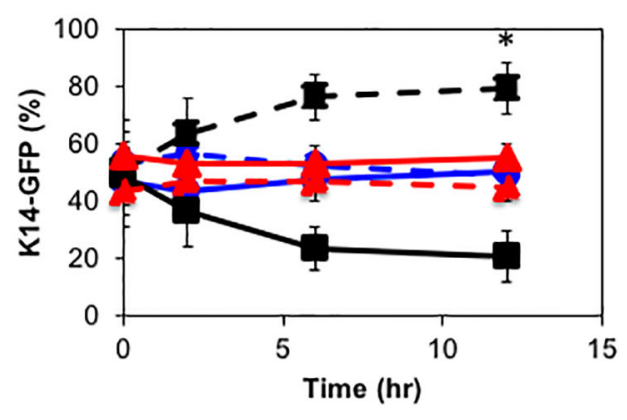

Figure 7: Primary tumor organoids migrate with flow when K14 leader cell polariz in the direction of flow.

(A) Time-lapse images of K14-GFP MMTV-PyMT (WT) and K14-GFP DDR2-/- MMTVPyMT (DDR2-/-) primary organoids in response to interstitial fluid flow under low oxygen conditions (arrows indicate K14-GFP cells; scale bar $=50 \mu \mathrm{m}$ ) (B) SHG imaging of remodeled collagen (C-D) Collective migration efficiency and average velocity (E) K14GFP fluorescence localization within tumor organoids (for all experiments: *p $<0.05$, ANOVA with Tukey's post-hoc analysis; $5 \% \mathrm{O}_{2}$ conditions) 